ARTICLE

\title{
Identification of FOXM1 as a therapeutic target in B-cell lineage acute lymphoblastic leukaemia
}

Maike Buchner ${ }^{1}$, Eugene Park ${ }^{1,2}$, Huimin Geng ${ }^{1}$, Lars Klemm¹, Johanna Flach ${ }^{3}$, Emmanuelle Passegué ${ }^{3}$,

Hilde Schjerven ${ }^{1}$, Ari Melnick ${ }^{4}$, Elisabeth Paietta ${ }^{5}$, Dragana Kopanja ${ }^{6}$, Pradip Raychaudhuri ${ }^{6}$ \&

Markus Müschen 1,2

Despite recent advances in the cure rate of acute lymphoblastic leukaemia (ALL), the prognosis for patients with relapsed ALL remains poor. Here we identify FOXM1 as a candidate responsible for an aggressive clinical course. We show that FOXM1 levels peak at the pre-B-cell receptor checkpoint but are dispensable for normal B-cell development. Compared with normal B-cell populations, FOXM1 levels are 2- to 60-fold higher in ALL cells and are predictive of poor outcome in ALL patients. FOXM1 is negatively regulated by FOXO3A, supports cell survival, drug resistance, colony formation and proliferation in vitro, and promotes leukemogenesis in vivo. Two complementary approaches of pharmacological FOXM1 inhibition-(i) FOXM1 transcriptional inactivation using the thiazole antibiotic thiostrepton and (ii) an FOXM1 inhibiting ARF-derived peptide-recapitulate the findings of genetic FOXM1 deletion. Taken together, our data identify FOXM1 as a novel therapeutic target, and demonstrate feasibility of FOXM1 inhibition in ALL.

\footnotetext{
${ }^{1}$ Department of Laboratory Medicine, University of California San Francisco, San Francisco, California 94143, USA. ${ }^{2}$ Department of Haematology, University of Cambridge, Cambridge CB2 OAH, UK. ${ }^{3}$ The Eli and Edythe Broad Center for Regenerative Medicine and Stem Cell Research, Department of Medicine, Hem/Onc Division, University of California San Francisco, San Francisco, California 94143, USA. ${ }^{4}$ Division of Hematology and Oncology, Weill Cornell Medical College, New York, New York 10021, USA. ${ }^{5}$ Montefiore Medical Center, Albert Einstein College of Medicine, Bronx, New York 10466, USA. ${ }^{6}$ Department of Biochemistry and Molecular Genetics, University of Illinois at Chicago, Chicago, Illinois 60607, USA. Correspondence and requests for materials should be addressed to M.B. (email: maike.buchner@ucsf.edu).
} 
F OXM1 belongs to the forkhead box transcription factor family and is a key regulator of cell growth by promoting cell cycle progression ${ }^{1}$. Expression of the FOXM1 protein is low in quiescent cells. During re-entry into the cell cycle, FOXM1 is expressed at late $\mathrm{G}_{1}$ /early $\mathrm{S}$-phase, sustained throughout the $\mathrm{G}_{2}$ phase and mitosis and its activity is regulated via phosphorylation ${ }^{2-4}$. This phosphorylation relieves it from its autoinhibitory conformation and allows it to drive the expression of additional cell cycle promoting molecules, such as Cdc25A as well as Skp2 and Cks1 (refs 5, 6) FOXM1 expression levels remain elevated in the $\mathrm{G}_{2^{-}}$and $\mathrm{M}$-phase, inducing the transcription of cyclin B1 (CCNB1), Aurora B kinase $(A U R K B)$ and Polo-like kinase 1 (PLK1) that are responsible for mitotic progression, spindle formation and chromosome segregation. Besides its role in cell cycle progression, FOXM1 has also been described to support cell survival through regulation of the antioxidant defense machinery of the cell, for example, by upregulating superoxide dismutase and Catalase (Cat) expression ${ }^{7}$.

Overexpression of FOXM1 has been implicated in the progression and drug resistance in a wide range of solid tumours 8 including liver ${ }^{9,10}$, colon $^{11}$, lung ${ }^{12-14}$ and prostate carcinoma ${ }^{15}$. Several studies highlight the function of FOXM1 in promoting cell proliferation, migration, angiogenesis and drug resistance that contributes to tumour initiation, growth and progression using transgenic mice as well as FOXM1 inhibitors ${ }^{8-21}$. Inducible deletion of Foxm1 in mouse models for lung adenomas, colon adenocarcinomas and hepatocellular carcinoma resulted in a significant reduction in tumorigenic potential and cancer cell proliferation ${ }^{10-14}$. A functional role of FOXM1 in haematopoietic malignancies has been suggested but further experimental validation is required for understanding the mechanism underlying its expression and contribution to disease progression ${ }^{16}$.

Despite advances in the cure rate of childhood pre- $B$ acute lymphoblastic leukaemia (ALL), the prognosis in older patients and for patients who experienced ALL relapse remains poor ${ }^{22}$. Philadelphia chromosome-positive $\left(\mathrm{Ph}^{+}\right)$ALL cells are driven by the oncogenic kinase BCR-ABL1 that arises from the $t(9 ; 22)(q 34 ; q 11)$ chromosomal translocation in pre-B cells. This subset has been associated with adult ALL and has a particularly poor clinical outcome $e^{23-25}$. The $B C R-A B L 1$ kinase can be specifically targeted by small-molecule tyrosine kinase inhibitors (TKIs) such as imatinib ${ }^{26}$. However, in contrast to BCR-ABL1driven chronic myeloid leukaemia (CML), $\mathrm{Ph}^{+}$ALL patients will invariably relapse after a short interval of remission, and develop TKI-resistant disease ${ }^{27}$.

Pre-B ALL emerges in virtually all cases from B-cell precursors that are arrested at the pre-B-cell receptor checkpoint. In a gene expression survey of early B-cell development, we found specific upregulation of FOXM1 at the pre-B-cell receptor checkpoint (Fig. 1a). Therefore, we investigate here the function of FOXM1 in normal B-cell development and in pre-B-cell-derived ALL with specific focus on its regulation and function in $\mathrm{Ph}^{+}$ALL. We reveal a FOXO3a-mediated transcriptional control of FOXM1 expression, a crucial function of FOXM1 with respect to TKI resistance and disease progression, using a conditional Foxm $1^{\mathrm{fl} / \mathrm{fl}}$ knockout mouse model as well as pharmacological inhibition of FOXM1 in patient-derived pre-B ALL cells.

\section{Results}

FOXM1 expression is dispensable in B-cell precursors. We found FOXM1 mRNA specifically upregulated at the pre-B-cell receptor checkpoint (Fig. $1 \mathrm{a})^{28}$. This was verified by quantitative real-time (qRT) PCR of sorted human B-cell precursors as well as murine B-cell progenitor fractions (Fig. 1b,c; sorting strategies and reanalysis of the sort are shown in Supplementary Fig. 1) ${ }^{28,29}$. To determine a potential function of FOXM1 in normal $B$ lymphopoiesis, we harvested bone marrow (BM) of a Foxm1 conditional knockout mouse model $\left(\text { Foxm } 1^{\mathrm{fl} / \mathrm{fl}}\right)^{20}$. B-cell precursors of Foxm $1^{\mathrm{fl} / \mathrm{fl}}$ mice were cultured in interleukin 7 (IL-7)-containing media and transduced with a 4-hydroxy tamoxifen (4-OHT)-inducible Cre-ER ${ }^{\mathrm{T} 2}$ (Cre) or empty vector $\left(\mathrm{EV} ; \mathrm{ER}^{\mathrm{T} 2}\right)$. 4-OHT-mediated deletion of Foxm1 did not significantly alter the viability of normal B-cell precursors (Fig. 1d,e, respectively) and is therefore not required for survival of IL-7-dependent pro/pre-B cells. Next we sought to analyse a potential role of Foxm1 in vivo during normal B-cell development. To this end, we crossed Foxm $1^{\mathrm{fl} / \mathrm{fl}}$ mice with $\mathrm{Mbl}-\mathrm{Cr} \mathrm{e}^{\mathrm{tg} /}+\mathrm{mice}$ to induce deletion of Foxm1 in early B-cell progenitors ${ }^{30}$. BM from 6-8-week-old $M b 1-C r e^{\text {tg } /+}$ Foxm $1^{\mathrm{fl} / \mathrm{fl}}$ mice or $\mathrm{Mb1} \mathrm{1}^{+1+}$ wt (wild-type) Foxm $1^{\mathrm{fl} / \mathrm{fl}}$ littermates were analysed for Hardy fractions $\mathrm{A}-\mathrm{F}^{29}$. In addition, spleen, lymph nodes and peritoneal cavity were analysed for number of $B$ cells and their immunoglobulin light-chain expression $(\kappa$ and $\lambda)$. Despite the observed high expression of Foxm1 mRNA in the $\mathrm{C}^{\prime}$ and $\mathrm{D}$ fractions, Foxm1 deletion did not alter B-cell development (examples of flow cytometry plots are shown in Fig. 1f, further analysis is shown in Supplementary Fig. 2a-d). Also the ability of pre- $\mathrm{B}$ cells to differentiate into $\kappa$-light-chain producing immature B cells in vitro was not affected by B-cell-specific deletion of Foxm 1 (Fig. 1g). The confirmation of Foxm1 deletion is shown by immunoblot in Fig. 1h.

To further define whether Foxm1 expression is required for the proliferation and survival of uncommitted cells, we isolated BM cells from Foxm $1^{\mathrm{fl} / \mathrm{fl}}$ mice and cultured them in the presence of IL-3/IL-6/SCF to induce proliferation of $\mathrm{Lin}^{-} \mathrm{Sca}-1^{+} \mathrm{c}-\mathrm{Kit}^{+}$ (LSK)-like haematopoietic progenitor cells and transduced them with EV or inducible Cre-green fluorescent protein (GFP) ${ }^{31}$. We followed GFP expression and observed no change of growth behaviour on Foxm1 deletion in LSK progenitor cells (Supplementary Fig. 2e).

FOXM1 is highly expressed in ALL. To investigate a potential role of FOXM1 in transformed B cells, we used a mouse model for $\mathrm{Ph}^{+} \mathrm{ALL}$ : to this end, BM-derived B-cell precursors were cultured in the presence of IL-7 and transformed with a retroviral BCR-ABL1 expression vector (schematic shown in Supplementary Fig. 3a). De novo expression of BCR-ABL1 increased levels of Foxm1 compared to normal IL-7-dependent pre-B cells (Fig. 2a). We compared the expression levels in human $\mathrm{B}$-cell populations isolated from $\mathrm{BM}$ or peripheral blood of healthy donors with patient-derived pre-B ALL samples. All patient-derived samples used in this study are listed in Supplementary Table 1 and enrichment efficiency of CD19 ${ }^{+}$and CD $19^{+} \mathrm{CD}_{10}{ }^{+} \mathrm{B}$-cell populations is shown in Supplementary Fig. 3b,c. While FOXM1 protein expression levels were low in both BM-derived B-cell precursors and mature B cells, patientderived pre-B ALL samples revealed 2- to 60-fold higher FOXM1 protein levels compared with $\mathrm{B}$ cells or B-cell precursor populations $(P=0.0014$ and $P=0.0215$, respectively; Student's $t$-test; Fig. 2b,c). To define whether high FOXM1 expression was specific for defined ALL subsets, we compared the protein levels in $\mathrm{Ph}^{+}$ ALL samples and samples driven by other oncogenes, derived from childhood or adult ALL without observing significant differences (Supplementary Fig. 3d). To further define whether FOXM1 expression was induced by BCR-ABL1 kinase activity, we treated patient-derived $\mathrm{Ph}^{+}$ALL samples with TKI. Although we did not observe short-term effects, after $96 \mathrm{~h}$ of TKI treatment, FOXM1 levels were significantly downregulated (Fig. 2d). To ensure that the observed FOXM1 downregulation is not 
a

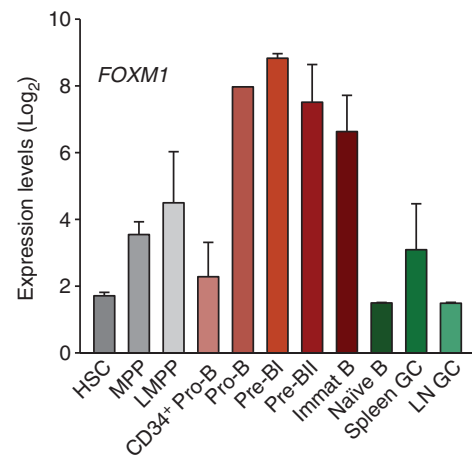

d

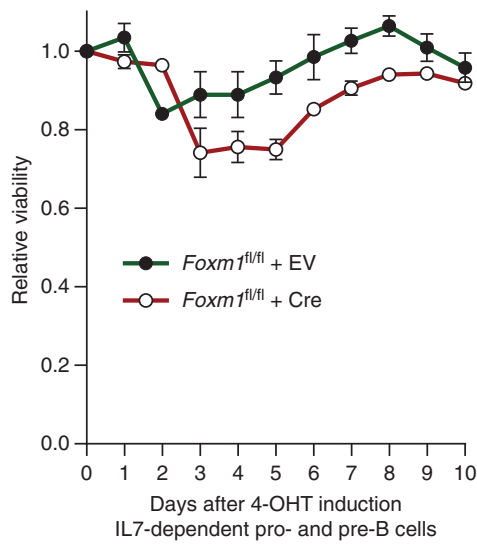

b

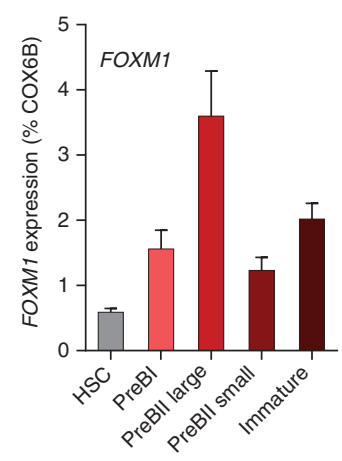

e

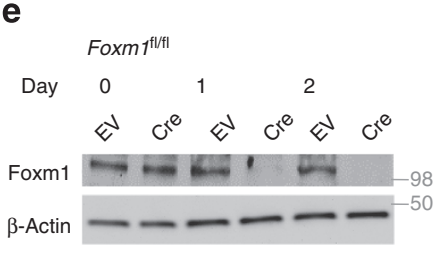

C

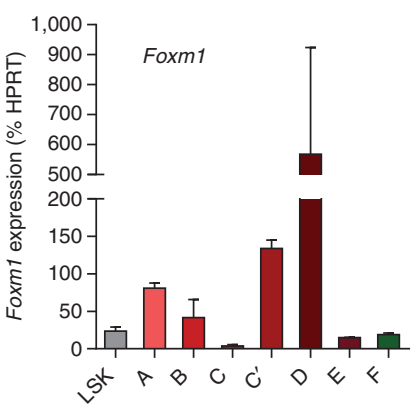

f Mb1wt Foxm $1^{\text {fl/fil }}$

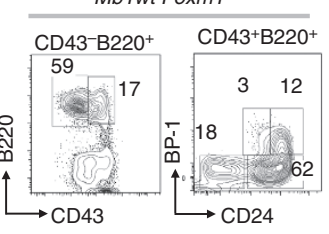

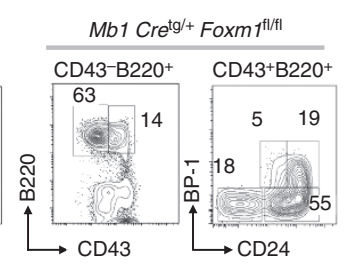

g

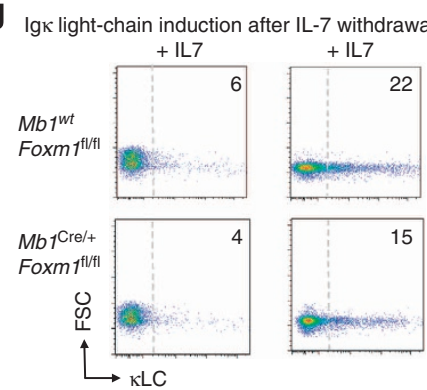

h

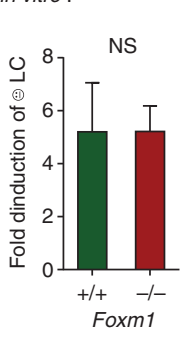
$\begin{array}{cc}\text { Mb1 } 1^{\mathrm{wt}} & M b 1^{\mathrm{Cre} /+} \\ \text { Foxm } 1^{\mathrm{fl} / \mathrm{fl}} & \text { Foxm1 }\end{array}$

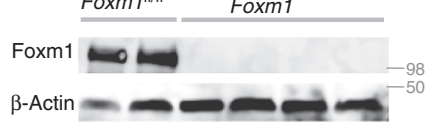

$\beta$-Actin $=$

Figure 1 | FOXM1 expression is dispensable for normal B-cell survival and development. (a) FOXM1 mRNA expression in sorted progenitor and B-cell fractions according to microarray data ${ }^{28}$ (b) qRT-PCR of FOXM1 in human B-cell progenitor fractions with COX6B used as a reference, mean values of $n=3$ (technical replicates); \pm s.e.m. (c) qRT-PCR of Foxm1 in Hardy B-cell fractions is shown with Hprt used as a reference, $n=3$ (technical replicates); \pm s.e.m.; representative example of two independent BM sorts. (d) Viability of Foxm $7^{\mathrm{fl} / \mathrm{fl}}$ B-cell precursors was analysed daily after induction of deletion by 4-OHT; representative results of three independent experiments are shown. (e) Immunoblot of Foxm1 deletion induced by $4-\mathrm{OHT}$ in Foxm $\mathrm{fl}^{\mathrm{f} / \mathrm{fl}} \mathrm{B}$-cell precursors. (f) Representative examples of Hardy Fraction A-C' for wt BM and Mb1-Cre ${ }^{\mathrm{tg} /}+\mathrm{Foxm}^{\mathrm{fl} / \mathrm{fl}}$ are shown. $(\mathbf{g}) \mathrm{BM}$ of $\mathrm{Mb} 1-\mathrm{Cre} \mathrm{tg}^{\mathrm{tg}}+\mathrm{Foxm} 7^{\mathrm{fl} / \mathrm{fl}}(\mathrm{n}=4)$ and $\mathrm{Mb1}$ wt Foxmi $7^{\mathrm{fl} / \mathrm{fl}}(n=3)$ littermates were cultured in the presence of IL-7. $\kappa \mathrm{LC}$ expression was induced by IL-7 withdrawal and analysed by flow

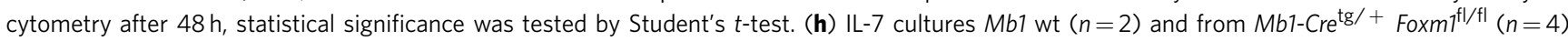
littermates were analysed for Foxm1 expression by immunoblot to confirm Cre-mediated Foxm1 deletion.

secondary to apoptosis induction but occurs as a consequence of BCR-ABL kinase inhibition, we overexpressed BCL2 in $\mathrm{Ph}^{+}$ALL cells and thereby abrogated apoptosis induction by $\mathrm{TKI}^{32}$. Similar to EV control cells, TKI treatment induced significant FOXM1 downregulation in the absence of apoptosis induction (Fig. 2e; corresponding viability is shown in Supplementary Fig. 3e). Interestingly, mitigating the effects of selection pressure (via BCL2 expression; Supplementary Fig. 3e) revealed a more rapid and even more profound downregulation of FOXM1 (Fig. 2e,f).

To further characterize the regulation of FOXM1, we analysed whether the inactivation of FOXO factors downstream of the $\mathrm{PI} 3 \mathrm{~K} / \mathrm{AKT}$ pathway contributes to high FOXM1 expression in $\mathrm{Ph}^{+} \mathrm{ALL}^{33-36}$. We overexpressed a constitutively active form of Akt (Myr-Akt) to prevent activation of FOXO factors in the presence of TKI. While this resulted in modest induction of Foxm1 expression in untreated cells, Myr-Akt expression entirely abrogated TKI-mediated downregulation of Foxm1 (Fig. 2g). To define whether Foxo3a is required for Foxm1 downregulation in ALL cells ${ }^{34}$, we generated Foxo3a-knockout BCR-ABL1-driven ALL and performed TKI treatment. Strikingly, in the absence of Foxo3a, FOXM1 levels remained high despite long-term TKI treatment (Fig. 2h). To further confirm the regulation of FOXM1 by FOXO3a, we overexpressed a constitutively active form of FOXO3a (FOXO3aAAA) ${ }^{37}$ and observed significant downregulation of Foxm 1 mRNA and protein levels compared with empty vector control (Fig. 2i,j). Active FOXO1, however, did not modulate FOXM1 levels on mRNA or protein levels (Supplementary Fig. 3f,g).

FOXM1 is a predictor of poor clinical outcome in ALL. To evaluate a potential predictive value of FOXM1 mRNA levels in patient-derived ALL samples at the time of diagnosis, we conducted a retrospective analysis of FOXM1 expression. First, we measured FOXM1 mRNA levels at the time of diagnosis in a clinical trial for patients with childhood ALL (BFM-REZ 2002). FOXM1 mRNA levels clearly correlated with risk stratification of childhood ALL and were significantly lower in BM biopsies from patients with intermediate-risk pediatric ALL $(n=31$; including one patient with $\mathrm{Ph}^{+}$ALL) compared with high-risk pediatric $\operatorname{ALL}\left(n=21\right.$; including one patient with $P h^{+}$ALL; $P=7.3 \mathrm{e}-5$, Wilcoxon rank-sum test). As a metric for ALL aggressiveness, 
a

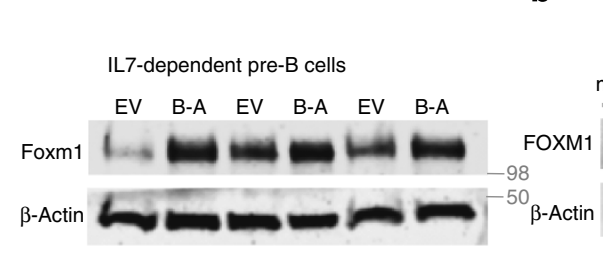

b

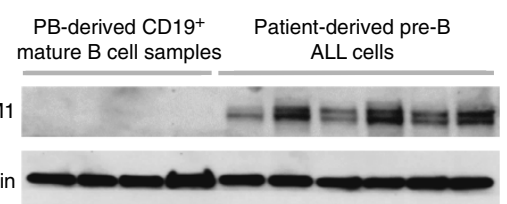

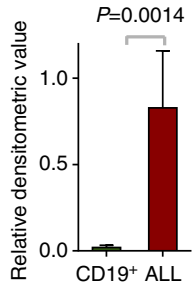

d c

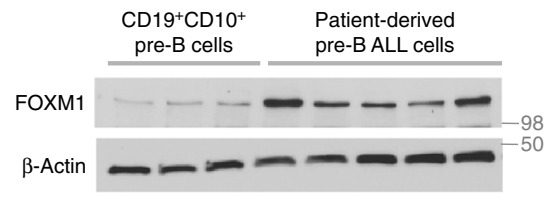

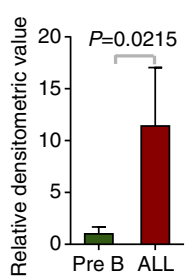

f e

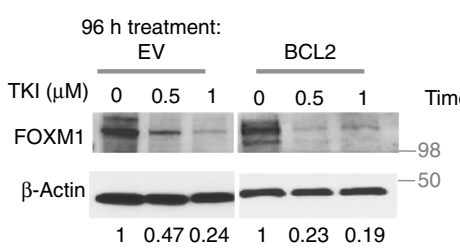

h

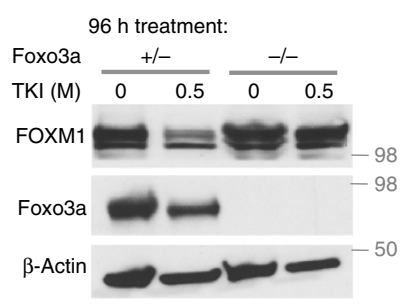

i

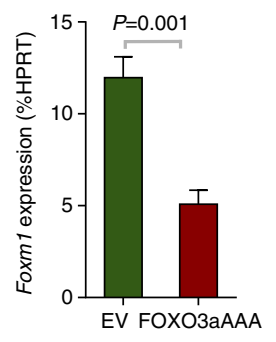

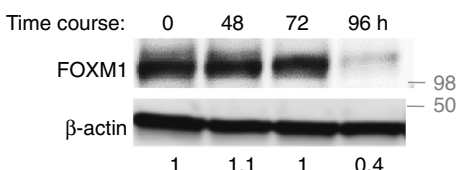

g

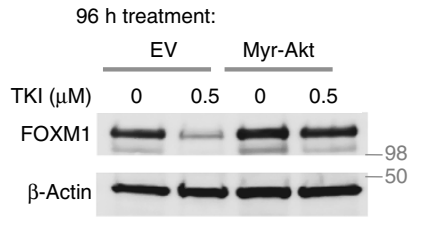

Figure 2 | Regulation of FOXM1 expression in patient-derived pre-B ALL cells. (a) Foxm1 expression levels of three matched samples of IL-7-dependent B-cell precursors (in the presence of IL-7) and BCR-ABL1-transformed ALL-like cells (IL-7 independent). (b,c) Immunoblot analysis for the expression of FOXM1 in normal CD19 ${ }^{+} \mathrm{B}$ cells $(\mathbf{b})$ derived from peripheral blood $(\mathrm{PB})$ or $\mathrm{CD} 19+\mathrm{CD} 10+\mathrm{B}$-cell precursors (c) derived from $\mathrm{BM}$ and patient-derived pre-B ALL; loading order for ALL: ICN1 $\left(P h^{+}\right)$, PDX2 $\left(P h^{+}\right)$, SFO5 (normal karyotype), LAX7 (normal karyotype), LAX7R (normal karyotype), LAX2 (Ph ${ }^{+}$) and PDX2 $\left(\mathrm{Ph}^{+}\right), \mathrm{MPX} 2\left(\mathrm{Ph}^{+}\right), \mathrm{MPX} 3\left(\mathrm{Ph}^{+}\right), \mathrm{MPX} 5\left(\mathrm{Ph}^{+}\right), \mathrm{BLQ} 5\left(\mathrm{Ph}^{+}\right)$, respectively; shown with $\beta$-actin as loading control. Mean densitometric values relative to loading control are shown in the bar graphs \pm s.e.m., significance was determined by Student's t-test. (d) Time course of FOXM1 protein levels after TKI treatment (shown for ICN1), $\beta$-actin was used as a loading control; representative result of three independent experiments is shown. (e) Human $\mathrm{Ph}^{+}$ALL cells (PDX2) were treated with TKI at low doses for $96 \mathrm{~h}$ and analysed for FOXM1 expression with $\beta$-actin as a loading control with and without overexpression of BCL2, representative result of three independent experiments is shown. (f) Time course analysis of FOXM1 expression in PDX2 with either empty vector or BCL2 overexpression. (g) Immunoblot analysis of Foxm1 with and without the expression of constitutively active Myr-Akt in murine ALL, treated with low concentrations of TKI or control for 96 h. (h) Immunoblot analysis Foxm1 expression in murine BCR-ABL1transformed Foxo3a $a^{+/-}$and Foxo3a- /- ALL treated with low concentrations of TKI or control for 4 days; representative result of three independent experiments is shown. (i) Foxm1 qRT-PCR was performed on day 2 after transduction with EV or a constitutively active form of FOXO3a (FOXO3aAAA), $H$ prt was used as a reference, $n=3$; \pm s.e.m., Student's $t$-test was used for statistical analysis. (j) Foxm1 protein levels were measured 3 days after the introduction of a constitutively active form of FOXO3a (FOXO3aAAA), with $\beta$-actin as a loading control.

FOXM1 mRNA expression levels also correlated with time to relapse (Fig. 3a; $n=52 ; P=0.00015$; Kruskal-Wallis rank-sum test). Consistent with data from the BFM-REZ 2002 trial, we found that FOXM1 mRNA levels are upregulated at the time of relapse in a pediatric high-risk ALL trial (COG P9906). Comparative analysis of FOXM1 mRNA levels from matched samples from 49 ALL patients at diagnosis and subsequent relapse revealed a significant upregulation of FOXM1 by the time of relapse $(P=3.8 \mathrm{e}-6$; paired $t$-test; Fig. $3 \mathrm{~b})$. In addition, we found a significant inverse correlation of FOXM1 with FOXO3A mRNA levels in $\mathrm{Ph}^{+}$ALL patients (Fig. 3c; Pearson correlation test) from the Medical Research Council UKALL XII/Eastern Cooperative Oncology Group (ECOG) E2993 trial. Overall survival (OS) analysis of this patient cohort revealed that a high ratio of FOXM1/FOXO3A was a predictor of poor OS in the high-risk subgroup of $\mathrm{Ph}^{+}$ALL (Fig. $3 \mathrm{~d} ; n=27 ; P=0.03$; Log-rank Mantle-Cox test). Analyses for the individual genes are shown in Supplementary Fig. 3h,i. Furthermore, a set of genes that is directly regulated by FOXM1 and associated with poor prognosis in breast cancer $^{18}$ showed significant correlation with poor 
a

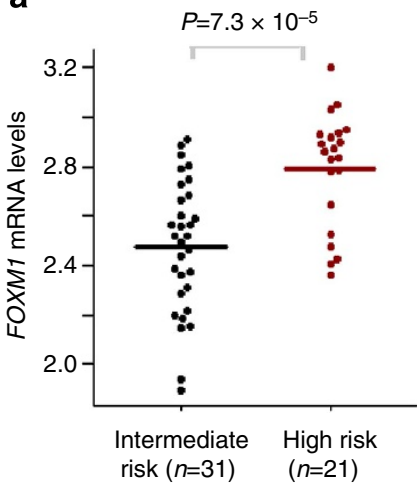

C

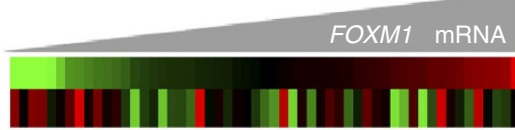

FOXM1

FOXO3A

$P=1.1 \times 10^{-6}$

Pearson's correlation $=-0.34$

d

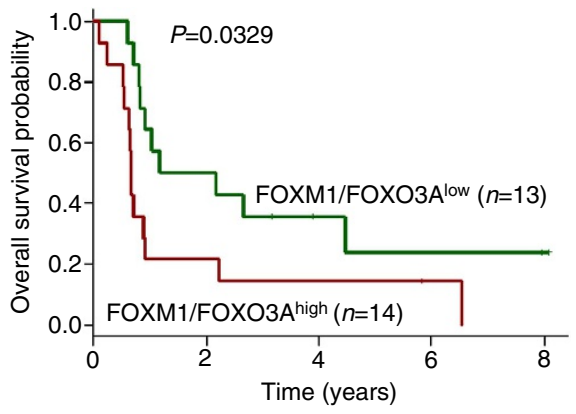

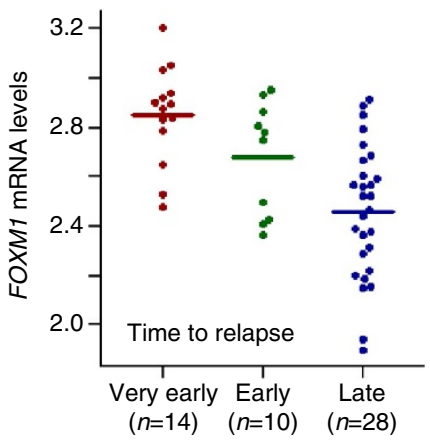

b

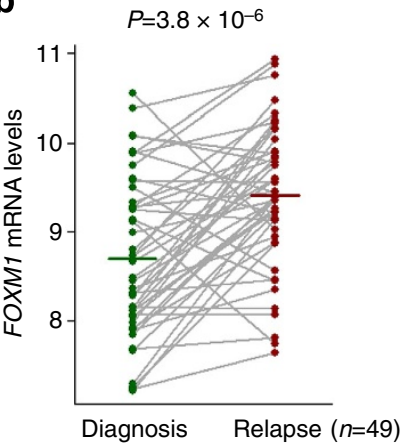

e

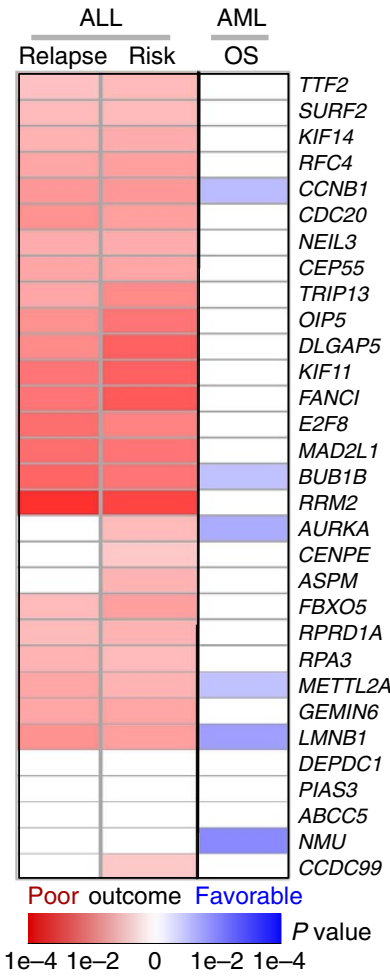

Figure 3 | FOXM1 is a predictor of poor clinical outcome in pre-B ALL. (a) Gene expression profiling of 60 cases of first relapse childhood ALL samples were analysed for FOXM1 expression and stratified into intermediate and high-risk groups according to a combination of prognostic factors of the relapse trial of the German Berlin-Frankfurt-Münster (BFM) study group ALL-REZ BFM 2002 (left panel; Wilcoxon rank-sum test was used for statistical analysis). Analysis of the same data set was correlated with time to relapse: very early relapse (within 18 months after initial diagnosis; $n=14$ ), early relapse ( $>18$ months after initial diagnosis but $<6$ months after cessation of frontline treatment; $n=10$ ) and late relapse ( $>6$ months after cessation of frontline treatment; $n=28$; right panel; Kruskal-Wallis rank-sum test was used for statistical analysis). (b) FOXM1 expression as determined by the gene expression profiling of matched samples of ALL at time of diagnosis and relapse. (c) Inverse correlation of FOXM1 with FOXO1A and FOXO3A mRNA levels in patients with $\mathrm{Ph}^{+} \mathrm{ALL}$ in the ECOG E2993 trial ( $n=55$; Pearson correlation test). (d) Patients with $\mathrm{Ph}^{+}$ALL in the ECOG E2993 trial $(n=55)$ were segregated into two groups based on higher or lower than median ratio of mRNA levels of FOXM1/FOXO3A. OS of patients in the FOXM1/FOXO3A ${ }^{\mathrm{Hi}}$ versus FOXM1/FOXO3A ${ }^{\text {Low }}$ group was compared by Kaplan-Meier analysis with the $P$ value determined by Log-rank Mantle-Cox test. (e) FOXM1 target genes $^{18}$ on a data set collected by the German ALL-REZ BFM 2002 of the BFM study group $(n=60)^{60}$ and AML (data retrieved from The Cancer Genome Atlas). A colour scale indicates whether the expression level of a gene is significantly associated with poor (in red colour) or favourable (in blue colour) patient outcome or not significant (in white colour), based on the parameters of relapse, risk stratification (as determined by Wilcoxon rank-sum test) and OS (as determined by Log-rank Mantle-Cox test).

clinical outcome in B-cell lineage ALL patients but not in myeloid lineage AML (Fig. 3e). In summary, these findings indicate that FOXM1 mRNA expression levels are associated with poor clinical outcome in ALL and inversely correlate with mRNA of FOXO3a.

Foxm1 mediates proliferation and survival of leukaemia cells. To further analyse the function and regulation of FOXM1 in pre-
B ALL cells, we focused our analysis on BCR-ABL1-driven ALL as a model for high-risk ALL and transformed B-cell precursors from Foxm $1^{\mathrm{fl} / \mathrm{fl}}$ mice with BCR-ABL1 and then transduced these with a 4-OHT-inducible Cre $\left(\mathrm{Cre}-\mathrm{ER}^{\mathrm{T} 2}\right)$ or an empty vector (EV; $\mathrm{ER}^{\mathrm{T} 2}$ ). Immunoblot analysis revealed a rapid deletion of Foxm1 after 4-OHT treatment (Fig. 4a), which induced cell death in a fraction of BCR-ABL1 +, ALL cells (Fig. 4b,c; flow cytometric analysis is shown in Supplementary Fig. 4a). To rule out 
a

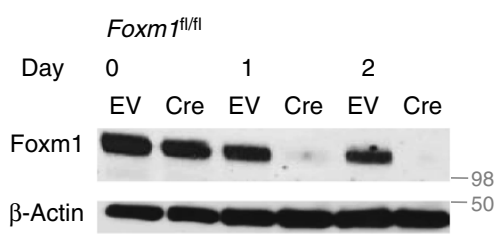

b

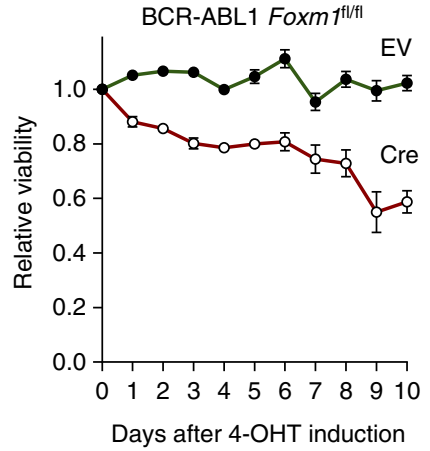

c

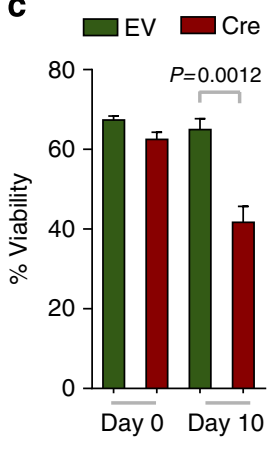

d
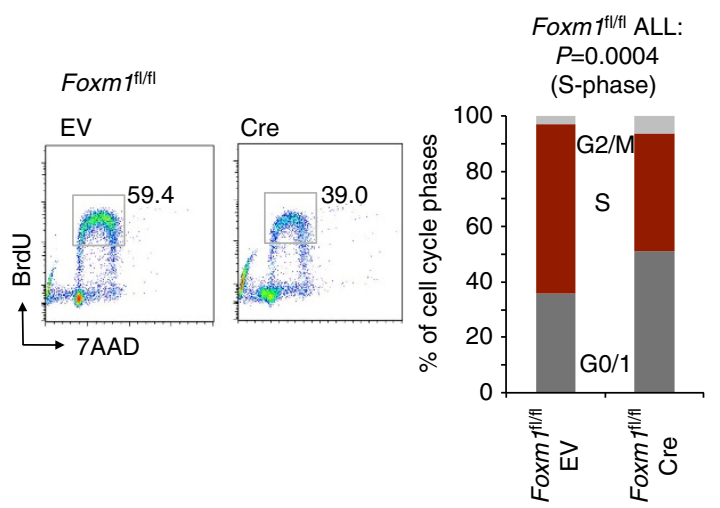

e

ALL: colony formation assay Foxm $1^{\text {fl/fl }}+\mathrm{EV}$ :

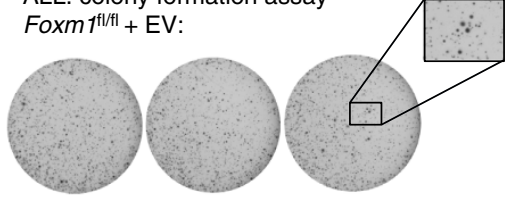

Foxm $1^{\mathrm{fl} / \mathrm{fl}}+$ Cre:
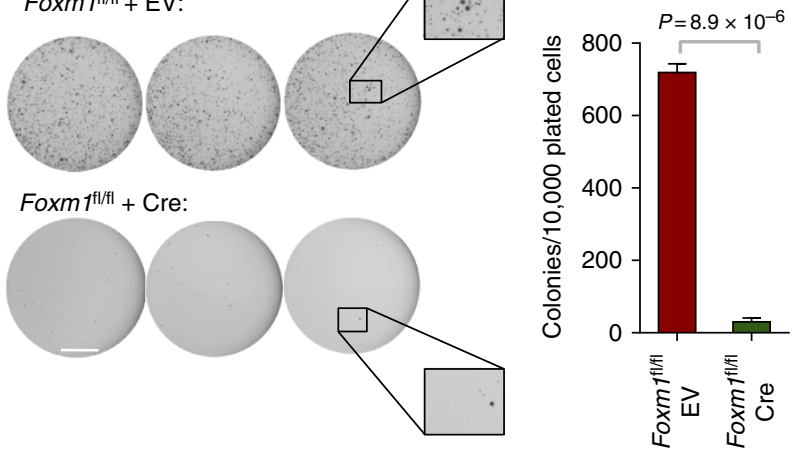

f

Survival of mice after injection of 100,000 $\mathrm{Ph}^{+} \mathrm{ALL}-$ like cells ( $n=7$; ex vivo deletion)

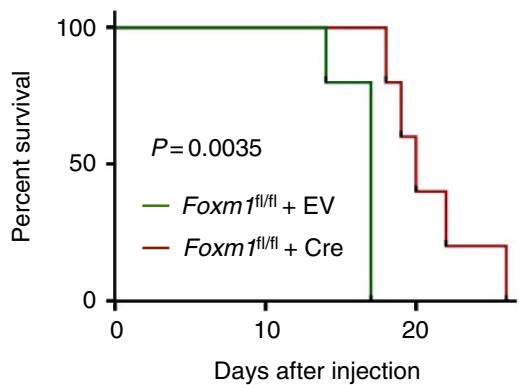

g

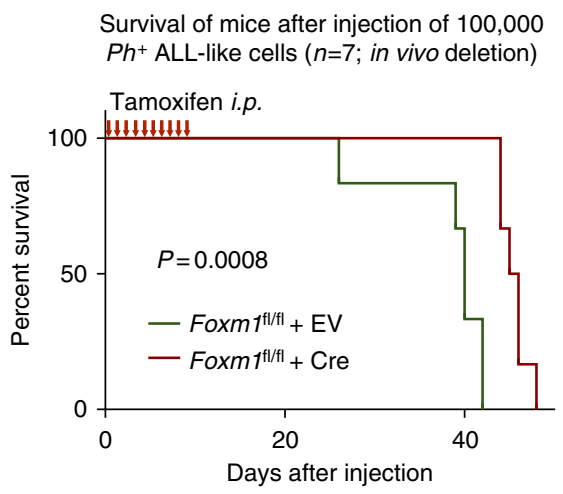

h

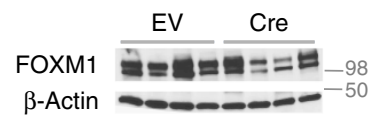

i

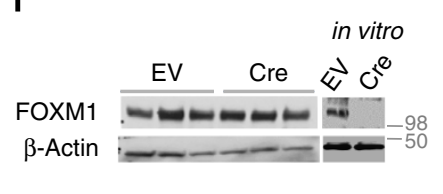

Figure 4 | Foxm1 mediates the proliferation and survival of leukaemia cells in a mouse model of $\mathbf{P h}{ }^{+}$ALL. (a) Deletion of Foxm1 in Foxm $7^{f l / f \mid} \mathrm{ALL}$ cells is shown by immunoblot with $\beta$-actin as a loading control. (b) Viability of ALL cells after induction of deletion was measured daily by flow cytometry (fluorescence-activated cell sorting blots are shown in Supplementary Fig. 4a; representative example of three independent experiments). (c) Comparison of viability in Foxm $7^{f l / f l} \mathrm{EV}$ and $\mathrm{Cre}$ on day 0 and day 10 after 4-OHT, $n=3 ; \pm$ s.e.m., Student's $t$-test was used for statistical analysis. (d) Cell cycle progression was measured based on BrdU incorporation and DNA content (7 AAD) after 2 days of 4-OHT treatment, $n=3$ (technical replicates); \pm s.e.m., Student's $t$-test was used for statistical analysis; representative example of four independent experiments. (e) $20,000 \mathrm{Foxm} 7^{\mathrm{fl} / \mathrm{fl}} \mathrm{EV}$ and Cre ALL

cells were plated in methylcellulose for 20 days in the presence of $4-\mathrm{OHT}$, colonies formed were counted; $n=3 ; \pm$ s.e.m., Student's $t$-test was used for statistical analysis, scale bar represents $1 \mathrm{~cm}$, squared section is twofold enlarged. (f) Kaplan-Meier analysis of NOD/SCID mice that were injected with

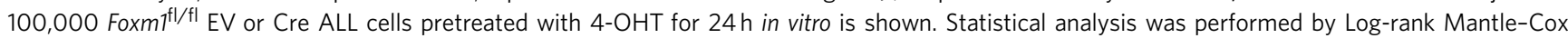
test. (g) Kaplan-Meier analysis of NOD/SCID mice that were injected with 100,000 Foxm $7^{\mathrm{fl} / \mathrm{fl}} \mathrm{EV}$ or Cre ALL cells and treated for 10 days with tamoxifen in corn oil i.p. is shown, $n=7$ per group. Statistical analysis was performed by Log-rank Mantle-Cox test. (h,i) Immunoblot revealed normal levels of Foxm 1 after ex vivo and in vivo deletion (indicating outgrowth of clones with incomplete deletion); complete deletion in vitro in pre-B cells in the presence of 4-OHT is shown for day 14 (i).

unspecific effects of Cre-mediated deletion in murine ALL, we also transduced Foxm1 ${ }^{+/+}$cells with EV and Cre and followed survival without observing any effect (Supplementary Fig. 4b). However, deletion of Foxm 1 resulted in arrest in both $\mathrm{G}_{0} / \mathrm{G}_{1}$ and $\mathrm{G}_{2} / \mathrm{M}$ phase with a significant reduction of the S-phase of the cell cycle (Fig. 4d) and colony formation in vitro was nearly entirely abolished by deletion of Foxm1 (Fig. 4e). To investigate whether Cre-mediated deletion of Foxm1 affects the course of leukaemia development in vivo, we injected 100,000 leukaemia cells carrying 4-OHT-inducible Cre or an empty vector control that were 
pretreated with 4-OHT in vitro for $24 \mathrm{~h}$ intrafemorally into sublethally irradiated NOD/SCID mice. Inducible deletion of Foxm1 significantly prolonged survival as shown in the KaplanMeier survival curve (Fig. 4f; $n=7$ per group; $P=0.0035$; Logrank Mantle-Cox test), without alteration of the ALL phenotype (representative phenotype shown in Supplementary Fig. 4c). Although we observed complete deletion of Foxm1 in vitro (Fig. 4a), ex vivo deletion and expansion of cell numbers in vivo revealed high levels of Foxm1 by the time of BM harvest from leukaemia-bearing mice, implying proliferative advantage of cells that evaded deletion of Foxm1 (Fig. 4h). In addition, we deleted Foxm1 in vivo by treating mice with tamoxifen after injection of 100,000 leukaemia cells carrying tamoxifen-inducible Cre or an empty vector control. We observed that deletion of Foxm1 significantly prolonged survival (Fig. $4 \mathrm{~g}$ ). However, similar to ex vivo deletion, clones that escaped deletion outgrew and formed lethal leukaemia (Fig. 4i). The presence of the floxed allele in these cells is confirmed by PCR, shown in Supplementary Fig. 4d. Collectively, these findings indicate that Foxm 1 plays a critical role in driving ALL cells proliferation, survival and leukaemia progression. The selective outgrowth of clones that escaped Cre-mediated deletion of Foxm1 further underscores the relevance of Foxm1 in the development of fatal ALL in vivo.

Foxm1 mediates TKI resistance by inducing Cat in ALL. As Foxm1 is a critical regulator of oxidative responses ${ }^{6}$, we analysed the intracellular reactive oxygen species (ROS) formation in the presence and absence of Foxm1 in BCR-ABL1-transformed ALL cells and observed consistently higher ROS levels after Foxm 1 deletion (Fig. 5a) but not in Cre-transduced Foxm1 ${ }^{+/+}$cells (Supplementary Fig. 5a). To elucidate the underlying mechanism of this effect, we analysed the mRNA expression of the several ROS scavengers described as Foxm 1 targets by $\mathrm{qRT}^{-\mathrm{PCR}^{6}}$ and found significantly reduced mRNA (Fig. 5b) and protein levels (Fig. 5c) of Cat after inducible Cre-mediated Foxm1 deletion. Cat rapidly degrades $\mathrm{H}_{2} \mathrm{O}_{2}$ to $\mathrm{O}_{2}$ and $\mathrm{H}_{2} \mathrm{O}$ and therefore helps to prevent oxidative damage ${ }^{38}$. To verify direct CAT regulation by FOXM1 in patient-derived $\mathrm{Ph}^{+}$ALL, we performed single-locus chromatin immunoprecipitation (ChIP) analysis of a known binding site of FOXM1 in intron 1 of the CAT gene ${ }^{6}$. This revealed specific binding of FOXM1, albeit low ChIP enrichment compared with the positive control CCNB1 (Fig. 5d) ${ }^{1,6}$. The $A C T A$ gene served as a negative control as well as an unrelated region in the $C C N B 1$ gene promoter.

Interestingly, we found that Cat expression is increased in ALL cells after TKI treatment at the mRNA and protein levels (Fig. 5e,f). This suggests that low-dose TKI treatment may initially induce ROS formation, comparable to ROS induction following growth factor deprivation ${ }^{39}$, which usually is compensated by a functional antioxidant response machinery, for example, by Cat expression. To evaluate whether the observed Cat upregulation by TKI is Foxm1 dependent, we treated ALL cells with TKI and evaluated Cat expression in the presence and absence of Foxm1. Only the Foxm1-expressing ALL cells $\left(\right.$ Foxm $\left.1^{\mathrm{fl} / \mathrm{fl}}+\mathrm{EV}\right)$ were capable of upregulating Cat after $4 \mathrm{~h}$ TKI treatment (Fig. 5f). On the basis of these findings and the observed selective advantage of FOXM1-expressing cells in the presence of TKI we had observed (Fig. 2e,f), we further investigated the function of FOXM1 in mediating TKI resistance and determined TKI responsiveness of $B C R-A B L 1$-driven ALL cells in the presence and absence of Foxm1. On deletion of Foxm1, ALL cells revealed a strikingly higher sensitivity towards TKI treatment, particularly in response to treatment with very low TKI doses. For Foxm $1^{\text {fl/fl }}$ ALL cells, an $\mathrm{IC}_{50}$ of $325 \mathrm{nmoll}^{-1}$ was measured for imatinib compared with an $\mathrm{IC}_{50}$ of
$75 \mathrm{nmoll}^{-1}$ for Foxm1 ${ }^{-1-}$ ALL cells (Fig. 5g, closed circles). This was verified by Annexin $\mathrm{V}$ staining in the presence and absence of imatinib, with and without Foxm1 deletion, respectively (Supplementary Fig. 5b). To define whether enforced expression of Cat can rescue the effect of Foxm 1 deletion, we overexpressed Cat in wt and Foxm $1^{-1-}$ cells and conducted TKI sensitivity analysis. While Cat overexpression did not alter TKI sensitivity in the presence of Foxm1, Foxm1deficient cells were significantly less sensitive in the presence of Cat as compared with EV control (Fig. 5g, open circles). Although, similar to FOXM1, FOXO3a may drive Cat transcription in the presence of TKI, we did not observe alterations in Cat expression in Foxo3a deficient ALL treated with TKI (Supplementary Fig. 5c) ${ }^{40}$.

FOXM1 inhibition induces apoptosis and TKI sensitivity. Our genetic experiments indicate that FOXM1 is particularly relevant for proliferation and survival of leukaemic B lymphoblasts but not normal B-cell development and survival and may, therefore, represent a tumour-specific therapeutic target. For this reason, we tested two complementary approaches of pharmacological inhibition of FOXM1 based on (i) an ARF-derived peptide that inhibits FOXM1 by sequestering it to the nucleolus and mediating its degradation and (ii) FOXM1 transcriptional inactivation using the thiazole antibiotic, thiostrepton.

Strategy I FOXM1 inhibition using a p19ARF-derived peptide. A previously described ARF peptide binds and inhibits FOXM1 function. The tumour suppressor p19ARF directly binds to FOXM1 and has been described to mediate its translocation to the nucleolus, which may result in its degradation ${ }^{10,36,41}$. The p19ARF amino acids 26-44 are sufficient to mediate the inhibitory function on FOXM1 and by addition of nine lipophilic arginines, the ARF-derived peptide can permeate across the plasma membrane $\left(\mathrm{ARF}_{26-44}\right)^{9,10}$. To verify the inhibitory effect on FOXM1, we treated patient-derived pre-B ALL cells with $\mathrm{ARF}_{26-44}$ and control peptide and analysed FOXM1 target gene transcription by qRT-PCR. As shown in Fig. 6a, we found significant inhibition of FOXM1 transcriptional activity after ARF peptide treatment. To further confirm the specificity, we treated Foxm1 $1^{-/-}$cells with ARF peptide concentrations up to $30 \mu \mathrm{M}$ and found cells still unresponsive (Fig. 6b). To verify the described mechanism of FOXM1 translocation induced by the ARF peptide, we performed immunostaining of nucleoli with Fibrillarin and costained with FOXM1. Despite the observed downregulation of FOXM1 target gene, we were not able to visualize the ARFmediated nucleolar localization of FOXM1 (Fig. 6c). However, in agreement with previous reports of ARF-targeted proteins that undergo nucleolar degradation, we observed the downregulation of FOXM1 protein levels in the presence on ARF peptide as a mechanism for FOXM1 inhibition (Fig. 6d ${ }^{42,43}$. To confirm the finding of apoptosis induction in our genetic deletion studies of Foxm1, we treated patientderived ALL samples with various amounts of ARF peptide and found significant growth inhibition after $72 \mathrm{~h}$ (Fig. 6e). B-cell lymphoma and myeloid lineage CML cells were treated in parallel experiments and were significantly less sensitive towards the $\mathrm{ARF}_{26-44}$ peptide. In addition, we confirmed that inhibition of FOXM1 by the $\mathrm{ARF}_{26-44}$ peptide sensitized patient-derived $\mathrm{Ph}^{+}$ALL cells towards TKI treatment compared with TKI treatment alone (Fig. 6f) and increased intracellular levels of ROS (Fig. 6g). To evaluate the effects of the ARF peptide on leukaemic cell growth in vivo, we injected 500,000 luciferaselabelled, patient-derived pre-B ALL cells into NSG mice and 
a

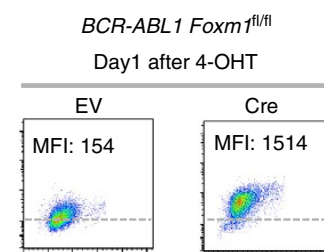

d2 after 4-OHT

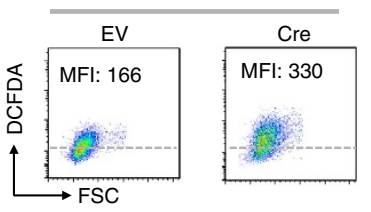

d
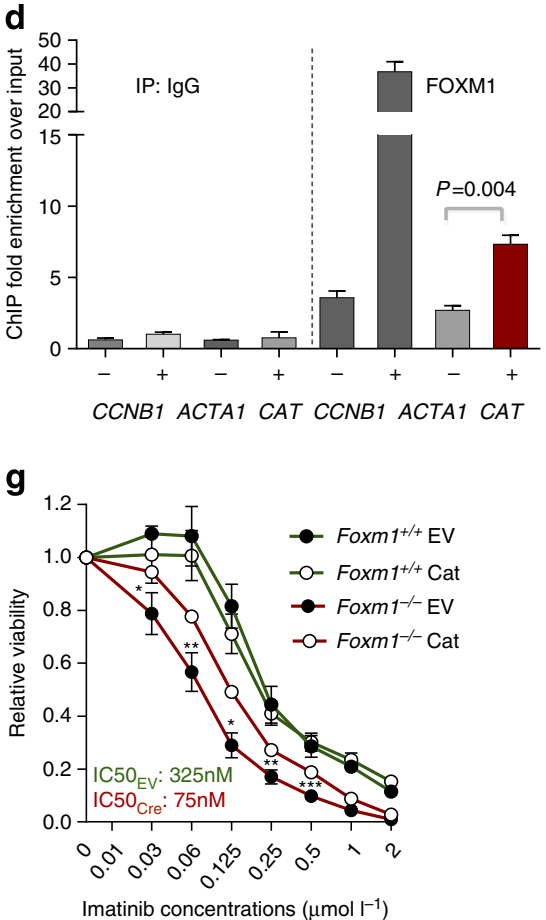

b

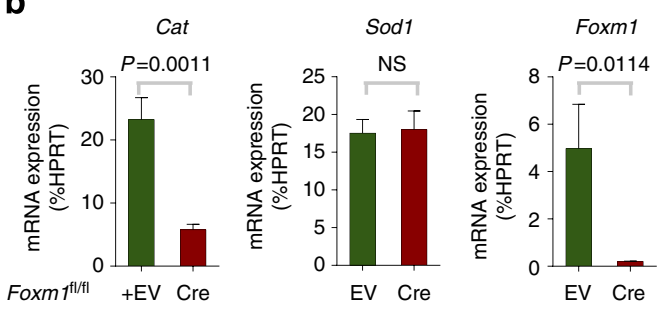

C Foxm $1^{\text {fl/fl }}+\mathrm{EV}$ Cre EV Cre EV Cre

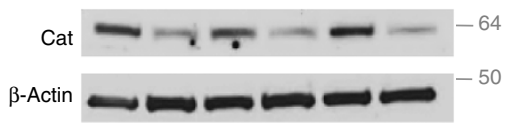

e

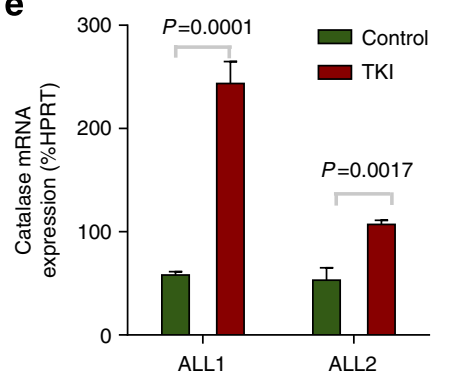

$\mathbf{f}$

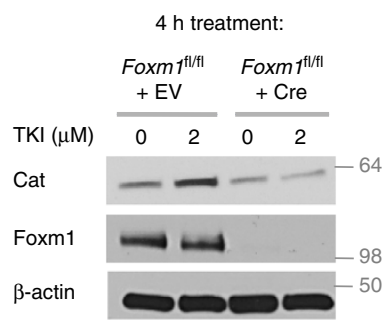

Figure 5 | Foxm1 enables resistance to TKIs in Ph ${ }^{+}$ALL. (a) Foxm $7^{f l / f l} E V$ or Cre ALL cells were stained with $2^{\prime} 7^{\prime}$-dichlorofluorescein diacetate (DCFDA), which labels cells based on intracellular levels of ROS after the indicated time of 4-OHT treatment. Mean fluorescence intensities (MFI) for DCFDA are depicted; $n=3$ (biological replicates); \pm s.e.m., Student's t-test was used for statistical analysis. (b) qRT-PCR analysis for Cat, Sod1 and Foxm1 mRNA levels in Foxm $7^{f l / f l} \mathrm{EV}$ or Cre ALL-like cells, Hprt is used as a reference, $n=3$; \pm s.e.m., Student's $t$-test was used for statistical analysis. (c) Immunoblot analysis of Cat $\mathrm{d} 2$ after 4-OHT in Foxm $7^{f l / f l} \mathrm{EV}$ or Cre ALL cells is shown. $\beta$-Actin serves as a loading control; samples are derived from three independent experiments. (d) Single-locus ChIP assay was performed on patient-derived ALL (PDX2) with normal rabbit IgG as a negative control. $(+)$ indicates a previously described binding domain of FOXM1, $(-)$ indicates the negative control, either within the same gene promoter or in the ACTA1 gene promoter; representative result of two independent experiments is shown. (e) Cat mRNA levels are measured after $4 \mathrm{~h}$ of imatinib treatment by quantitative real-time PCR with Hprt as a reference gene $n=3$; \pm s.e.m., Student's $t$-test was used for statistical analysis, two independent experiments are shown. (f) Cat expression levels are shown in the presence and absence of Foxm1, with and without imatinib treatment. (g) Imatinib (TKI)-sensitivity of BCR-ABL1transformed Foxm $7^{\mathrm{fl} / \mathrm{fl}} \mathrm{ALL}$ cells was measured in dose-response experiments after $72 \mathrm{~h}$, starting 2 days after $4-\mathrm{OHT}$ treatment. Relative values to untreated cells are displayed. Foxm $1^{-/-}$and Foxm $1^{+/+}$cells (EV; closed circles) are shown as well as overexpression of Cat for both conditions (open circles); representative result of three independent experiments is shown.

treated the mice with $10 \mathrm{mg} \mathrm{kg}^{-1} \mathrm{ARF}$ peptide on 10 consecutive days intravenously (i.v.) and intraperitoneally (i.p.), respectively, or vehicle control. This reduced the leukaemia burden as measured by luciferase bioimaging (Fig. 6h) and prolonged OS (Fig. 6i). Thus, the treatment results of patient-derived pre- $\mathrm{B}$ ALL samples with the $\mathrm{ARF}_{26-44}$ peptide (Fig. 6) confirmed our findings from the genetic deletion experiments in the ALL mouse model (Fig. 5) and demonstrate that peptide-based inhibition/degradation of FOXM1 protein induces selective toxicity in B-cell lineage ALL cells.
Strategy II transcriptional FOXM1 inhibition via thiostrepton. In a complementary approach, we evaluated the naturally occurring antibiotic thiostrepton (structure shown in Fig. 7a), which prevents FOXM1 from binding and activating its own promoter ${ }^{44}$. This autoregulatory positive feedback loop is critical for sustained FOXM1 expression levels ${ }^{45-48}$. Therefore, we tested whether thiostrepton was effective in suppressing FOXM1 protein levels in patient-derived ALL cells and reduction of FOXM1 target gene expression. Importantly, thiostrepton effectively disrupted the FOXM1's autoregulatory positive 
ARF $_{26-44}$ peptide RRRRRRRRRKFVRSRRPRTASCALAFVN Control peptide RRRRRRRRR-----------SCALAFVN

a
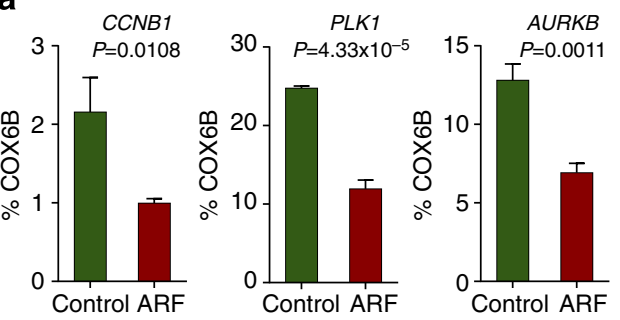

b

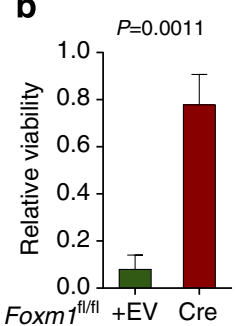

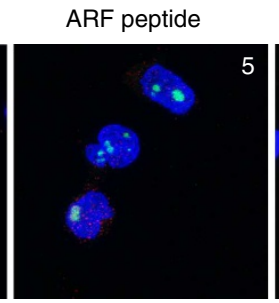

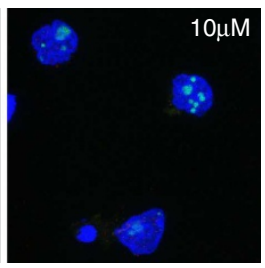

d

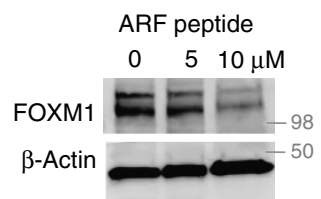

g

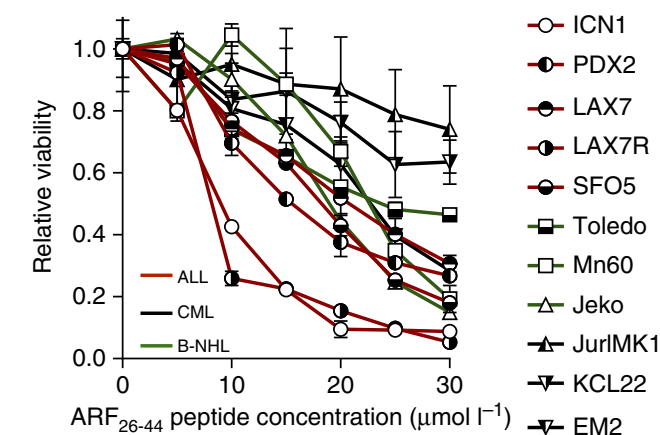

h
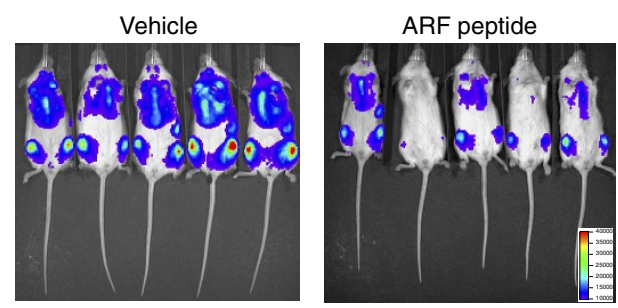

f

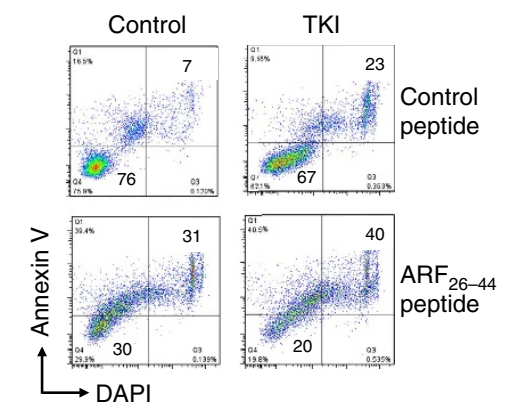

i

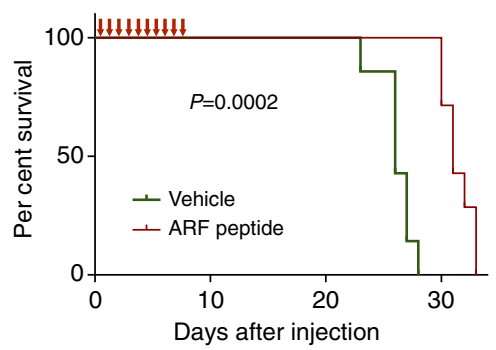

Figure 6 | In vivo efficacy of a peptide-based FOXM1 inhibitor in patient-derived ALL cells. (a) Analysis of FOXM1 target genes after $24 \mathrm{~h}$ of $10 \mu \mathrm{M}$ ARF peptide treatment of patient-derived ALL samples (shown for LAX2), COX6B was used as a reference gene, $n=3$; \pm s.e.m., Student's $t$-test was used for statistical analysis. (b) Efficacy of $30 \mu \mathrm{M}$ ARF peptide on Foxm ${ }^{+/+}$and Foxm $1^{-/-}$cells after $72 \mathrm{~h}$ to confirm specificity; measured by CCK-8 viability assay; $n=3$; \pm s.e.m., Student's $t$-test was used for statistical analysis. (c) Confocal microscopy of control or ARF peptide-treated ALL samples (shown for LAX7R), stained for the nucleoli marker fibrillarin (green) and FOXM1 (red) and 4',6-diamidino-2-phenylindole (DAPI). Scale bar represents $8 \mu \mathrm{m}$; representative result of three independent experiments. (d) FOXM1 protein expression after ARF peptide treatment after 24h (shown for LAX7R) (e) Dose-response for $\mathrm{ARF}_{26-44}$ peptide of pre-B ALL versus B-NHL (green) and CML cell lines (black), ICNI and PDX2 are Ph ${ }^{+}$ALL, SFO5, LAX7 and LAX7R are normal karyotype ALL (red). (f) Annexin V/DAPI staining after $48 \mathrm{~h}$ of $10 \mu \mathrm{M}$ imatinib in the presence of control peptide or ARF peptide of ICN1. (g) Intracellular ROS levels determined by DCFDA staining in ALL cells treated for $4 \mathrm{~h}$ with $10 \mu \mathrm{M}$ ARF peptide or control peptide (PDX2). (h) Leukaemia burden in mice after injection of 500,000 human luciferase-labelled pre-B ALL cells (LAX7R) and treatment for 10 days with ARF peptide, measured by luciferase bioimaging. (i) A Kaplan-Meier analysis compared overall survival of transplant recipients in the two groups; $n=7$ per group. Statistical analysis was performed by Log-rank Mantle-Cox test.

feedback loop and drastically attenuated FOXM1 expression, while the expression of other Forkhead box family members is not affected (Fig. 7b). The FOXM1 target genes CCNB1, PLK1 and $A U R K B$ mRNA levels were significantly reduced after Thiostrepton administration, confirming its reduced transcriptional activity (Fig. 7c). In addition, we treated Foxm $1^{-/}$- cells with Thiostrepton and observed these mainly unresponsive up to $0.5 \mu \mathrm{M}$ treatment for $72 \mathrm{~h}$, confirming its specificity towards FOXM1 at this concentration (Fig. 7d). We then tested whether the suppression of FOXM1 induced leukaemia-specific cell death in patient-derived pre-B ALL cells and observed significant cytotoxicity at concentrations of $0.5 \mu \mathrm{moll}^{-1}$ thiostrepton. By contrast, B-cell lymphoma and myeloid leukaemia cells were not responsive to thiostrepton treatment at similar concentrations (Fig. 7e). In accordance with similar levels of FOXM1 expression (Supplementary Fig. 3b), we did not observe differences in Thiostrepton sensitivity between pre-B ALL subsets in regards to childhood or adult ALL and $\mathrm{Ph}^{+}$or other ALL, respectively (Supplementary Fig. 5d). In addition, treatment with thiostrepton resulted in ROS accumulation and Cat regulation similar to what we had observed in the genetic deletion model of Foxm1: we treated Foxm1-expressing cells with thiostrepton for $4 \mathrm{~h}$ and analysed intracellular ROS levels in comparison with Foxm1 deletion ( $24 \mathrm{~h}$ after 4 -OHT). Similar increases of ROS were observed in the thiostrepton-treated Foxm1-expressing cells as in the Foxm $1^{-1-}$ ALL cells (Fig. 7f). To confirm the specific role of Foxm 1 in the increase of ROS formation, we included Foxm $1^{-/-}$ALL cells in the analysis. Thiostrepton treatment increased the formation of ROS only in the presence of Foxm1, 
a

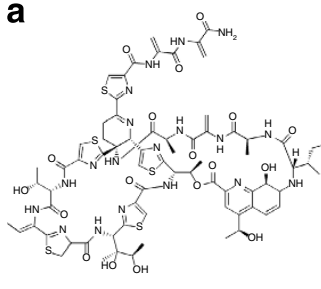

Thiostrepton (MW: 1,665)

d

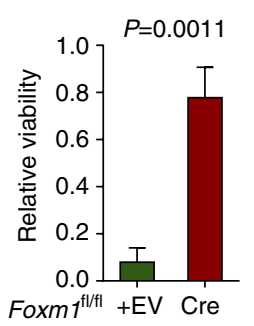

e b

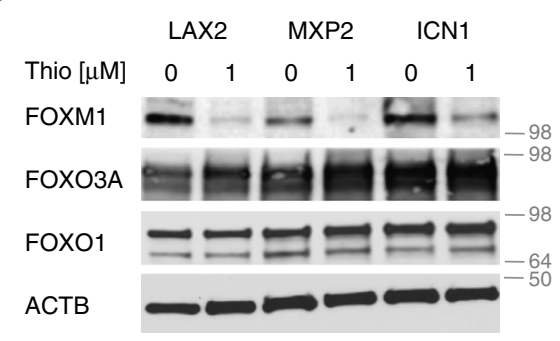

c

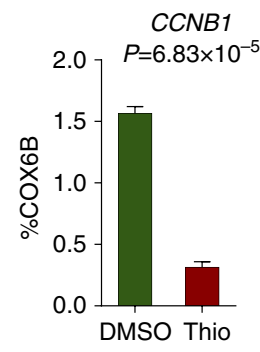

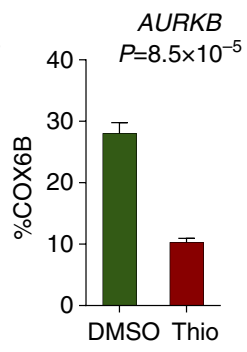

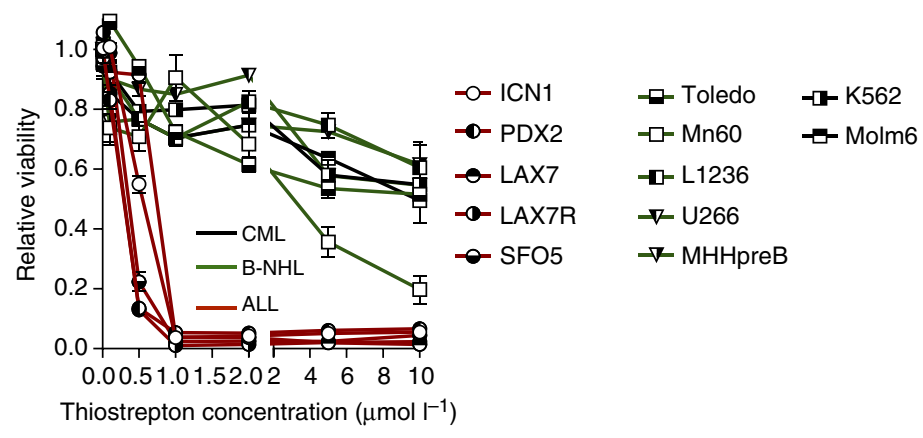

h f

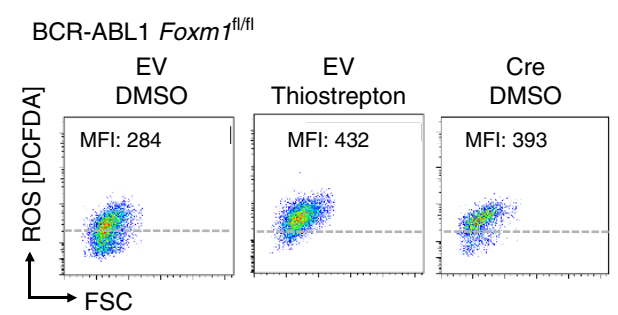

9

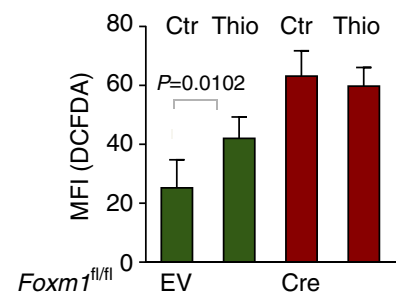

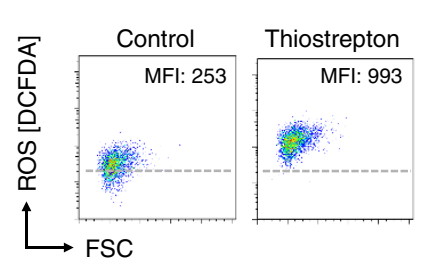

j
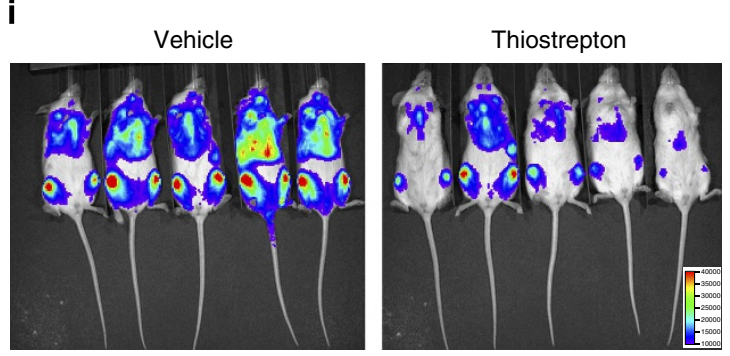

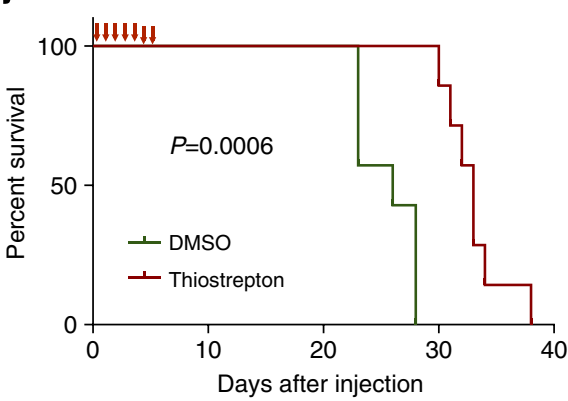

$\mathbf{k}$ In vivo $\left(50 \mathrm{mg} \mathrm{kg}^{-1}\right)$ : DMSO Thiostrepton

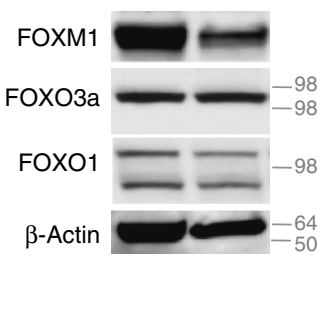

Figure 7 | In vivo efficacy of the FOXM1 inhibitor Thiostrepton in patient-derived ALL cells. (a) Thiostrepton structure is shown. (b) Immunoblot of FOXM1, FOXO3a, FOXO1 and $\beta$-actin as loading control after $18 \mathrm{~h}$ of treatment with thiostrepton at the indicated concentration. (c) Analysis of FOXM1 target genes after $24 \mathrm{~h}$ of $10 \mu \mathrm{M}$ ARF peptide treatment of patient-derived ALL samples (shown for LAX2). (d) Efficacy of $0.5 \mu \mathrm{M}$ Thiostrepton on Foxm $1^{+/+}$and Foxm $1^{-/-}$cells after $72 \mathrm{~h}$ to confirm specificity; measured by CCK-8 viability assay, $n=3 ; \pm$ s.e.m., Student's $t$-test was used for statistical analysis; representative result of three independent experiments. (e) Dose-response of pre-B ALL (red) versus B-cell lymphoma (green) or CML cell lines (black). (f) Intracellular ROS levels in Foxm $7^{f l / f l} \mathrm{EV}+\mathrm{DMSO}$ (vehicle) and Foxm $\mathrm{fl}^{\mathrm{fl} / \mathrm{fl}} \mathrm{EV}+$ thiostrepton after $4 \mathrm{~h}$. As a positive control Foxm $\mathrm{fl}^{\mathrm{f} / \mathrm{fl}} \mathrm{Cre}-\mathrm{ER} 4$ OHT ALL cells (day 1) was included. (g) ROS levels were determined in the presence and absence of Foxm 1 and with and without thiostrepton for $4 \mathrm{~h}$ with no further increase of ROS in the three independent Foxm1-deleted ALL-like cell lines, MFI levels are shown, $n=3 ; \pm$ s.e.m., Student's $t$-test was used for statistical analysis. (h) ROS induction in human patient-derived $\mathrm{Ph}^{+} \mathrm{ALL}$ cells (shown for PDX2) after $4 \mathrm{~h}$ of thiostrepton treatment is shown. (i) Leukaemia burden in mice after injection of 500,000 human luciferase-labelled pre-B ALL cells (LAX7R) and treated for seven consecutive days with 50 mg kg ${ }^{-1}$ Thiostrepton i.v., measured by luciferase bioimaging. (j) A Kaplan-Meier analysis compared the OS of transplant recipients in the two groups. Statistical analysis was performed by Log-rank Mantle-Cox test. (k) Immunoblot analysis of FOXM1, FOXO3a and FOXO1 in human xenografted ALL (LAX7R) harvested from mice showing signs of leukaemia, and then treated with three doses of thiostrepton at $50 \mathrm{mg} \mathrm{kg}^{-1}$ or vehicle control.

while the ROS levels in Foxm1-/- cells were not increased further by thiostrepton (Fig. $7 \mathrm{~g}$, representative example shown in Supplementary Fig. 5e). We subsequently verified Thiostrepton-mediated ROS formation in patient-derived $\mathrm{Ph}^{+}$
ALL after $4 \mathrm{~h}$ of treatment (Fig. 7h). To evaluate the effects of Thiostrepton in vivo, we injected 500,000 luciferase-labelled patient-derived pre-B ALL into NSG mice and treated the mice seven consecutive days i.v. with $50 \mathrm{mg} \mathrm{kg}^{-1}$ Thiostrepton daily 
or vehicle control. The daily treatment with Thiostrepton for 7 days reduced the leukaemia burden as measured by luciferase bioimaging (Fig. 7i) and prolonged OS (Fig. 7j). At the time point of harvest, the human origin of cells was confirmed by flow cytometry (representative example shown in Supplementary Fig. 5f). Confirming its biological activity in vivo, three doses of $50 \mathrm{mg} \mathrm{kg}^{-1}$ Thiostrepton i.v. effectively downregulated FOXM1 in xenografted ALL in vivo while it did not affect the expression of forkhead box transcription family members FOXO1 and FOXO3a (Fig. 7k).

\section{Discussion}

In this study, we have identified a critical function of the transcription factor FOXM1 in mediating proliferation and drug resistance in B-cell lineage ALL, but not in normal B-cell progenitors. Using two complementary approaches based on transcriptional inactivation and peptide-based inhibition of FOXM1, we demonstrate the feasibility and efficacy of FOXM1 inhibition in ALL.

Our data indicate that besides $\mathrm{Ph}^{+}$ALL, other subtypes of ALL with constitutive activation of FOXM1 downstream of AKT (for example, owing to the oncogenic activation of the Ras-MAPK pathway) are sensitive to FOXM1 inhibition as well and validate FOXM1 as a therapeutic target in a large fraction of drug-resistant B-cell lineage ALL. As a major factor in FOXM1-mediated drug resistance, we have identified the antioxidant molecule Cat. ROS are induced by oncogenic stimulation such as BCR-ABL1 kinase activity and drive signalling and proliferation (for example, by inhibiting phosphatase activity ${ }^{49}$. However, high levels of ROS also sensitizes cells to further oxidative stress that lead to mitochondrial DNA damage and lipid peroxidation that result in cell death ${ }^{50,51}$. Similar to the effects of growth factor withdrawal or STAT5 inactivation ${ }^{52,53}$, increased ROS levels on TKI treatment may contribute to TKImediated cell death and require immediate compensation by antioxidant molecules ${ }^{54,55}$. We have unravelled a particular role of FOXM1 in this antioxidant response as the overexpression of Cat in Foxm $1^{-1-}$ cells partially re-establishes TKI sensitivity but does not alter sensitivity in wt ALL cells (Fig. 5h). However, Cat overexpression does not completely rescue the deleterious effects of Foxm1 deletion, suggesting that additional FOXM1-mediated mechanisms are important. Therefore, FOXM1 inhibition is advantageous over substances that enhance intracellular ROS that have been previously suggested to cooperate with BCR-ABL1 inhibition ${ }^{56,57}$. Although the function of FOXM1 as a driver of antioxidant response has been described, this link to TKI resistance is novel and further studies are required to define whether this mechanism is specific to ALL or relevant in solid tumours treated with TKI as well. Despite the difficulties of targeting transcription factors by specific small molecules, recent efforts have identified a novel FOXM1 inhibitor in a library screen of 50,000 molecules ${ }^{58}$.

By mediating FOXM1 translocation to the nucleolus and thereafter inducing its degradation, the tumour suppressor ARF has been described as a negative regulator of FOXM1 (ref. 9). Interestingly, the gene that encodes ARF, CDKN2A, is frequently deleted in lymphoid, but not myeloid, BCR-ABL1-driven leukaemia. TKI treatment of $B C R-A B L 1$-driven CML patients is remarkably successful in inducing durable remissions, while monotherapy in $\mathrm{Ph}^{+}$ALL result in relapse with TKI refractory disease within a few months. Although CDKN2A deletions support enhanced cell survival in multiple ways (such as reduced $\mathrm{p} 53$ activation via $\mathrm{Mdm} 2$ overexpression $)^{59}$, the lack of negative regulation of FOXM1 may also contribute to the rapid TKI resistance development in ALL but not CML. In line with this, we have observed that CML cells are less sensitive to FOXM1 inhibition.

Taken together, our study highlights the importance of FOXM1 expression in pre-B ALL. Our findings of FOXM1 expression, itself and in combination with FOXO3a, as a potential marker for risk stratification of both childhood and adult, $\mathrm{Ph}^{+}$ ALL provide a robust basis for further, prospective analyses of FOXM1 in clinical trials. In addition, our data highlights the role of FOXM1 in driving the antioxidant response after TKI treatment as a crucial mechanism of TKI resistance. By genetic deletion and pharmacological inhibition, we provide a rationale for FOXM1 inhibition as a potential therapeutic intervention, alone or in synergistic combinations with TKIs for pre-B ALL and putatively other kinase-driven tumor cells that rely on a functional antioxidant response machinery.

\section{Methods}

Statistical analysis of clinical parameters. Gene expression microarray and patient outcome data were obtained from the GEO database accession number GSE5314 of the Eastern Cooperative Oncology Group (ECOG) Clinical Trial E2993 for adult B-ALL $(n=54)^{54}$, from GSE28460 of the Children's Oncology Group Clinical Trial P9906 for children B-ALL $(n=49 \text { pairs })^{55}$, from GSE4698 of the ALL-REZ BFM 2002 trial for children B-ALL $(n=52)^{60}$, from GSE1456 of the Karolinska Hospital Stockholm-Gotland breast cancer registry for breast cancer $(n=159)^{61}$ and from TCGA (https://tcga-data.nci.nih.gov/tcga/

dataAccessMatrix.htm) for AML $(n=184)$. The end points of the clinical data include OS, relapse-free survival, risk, time to relapse and expression levels in samples at the time of relapse versus expression levels at diagnosis (for 49 matched sample pairs from the Children's Oncology Group).

Pre-B and leukaemia cell culture. Patient samples (listed in Supplementary Table 1) were provided from the Departments of Hematology and Oncology, University Hospital Benjamin Franklin, Berlin, Germany and the USC Norris Comprehensive Cancer Center in compliance with Institutional Review Board regulations (approval from the Ethik-Kommission of the Charite, Campus Benjamin Franklin and the IRB of the University of Southern California Health Sciences Campus). Informed consent was obtained from all suspects. Leukaemia cells derived from BM aspirates of patients with pre-B ALL were xenografted into sublethally irradiated NOD/SCID mice via tail vein injection. After passaging, leukaemia cells were harvested and cultured on OP9 stroma cells in $\alpha$ minimum essential medium without ribonucleotides and deoxyribonucleotides (Invitrogen, Carlsbad, CA), supplemented with $20 \%$ fetal bovine serum (FBS), 2 mM L-glutamine, $1 \mathrm{mM}$ sodium pyruvate, $100 \mathrm{IU} \mathrm{ml}^{-1}$ penicillin and $100 \mathrm{mg} \mathrm{ml}^{-1}$ streptomycin. Cell lines used in this study are listed in Supplementary Table 2. Mouse $B C R-A B L 1$-transformed pre-B cells were cultured in IMDM (Invitrogen) with GlutaMAX containing $20 \% \mathrm{FBS}, 100 \mathrm{IU} \mathrm{ml}^{-1}$ penicillin, $100 \mu \mathrm{g} \mathrm{ml}^{-1}$ streptomycin and $50 \mu \mathrm{M} \beta$-mercaptoethanol. Normal mouse pre-B cells were cultured in the presence of $10 \mathrm{ng} \mathrm{ml}^{-1} \mathrm{IL}-7$. Murine LSK-like cells were cultured in the presence of $10 \mathrm{ng} \mathrm{ml}^{-1}$ recombinant mouse IL-3, $25 \mathrm{ng} \mathrm{ml}^{-1}$ recombinant mouse IL-6 and $50 \mathrm{ng} \mathrm{ml}^{-1}$ recombinant mouse SCF (PeproTech).

Retroviral constructs and transduction. Transfection of MSCV-based retroviral constructs encoding BCR-ABL1(p210)-IRES-Neo, Cre-ER ${ }^{\mathrm{T} 2}-\mathrm{Puro}, \mathrm{ER}^{\mathrm{T} 2}$-Puro, Cre-GFP, Luciferase-GFP, CD90-FOXO3a(AAA; D.A. Fruman, University of California Irvine, Irvine, CA) and empty vector controls were performed using Lipofectamine 2000 (Invitrogen) with Opti-MEM media (Invitrogen). Retroviral supernatant was produced by co-transfecting HEK 293FT cells with the plasmids pHIT123 (ecotropic env) and pHIT60 (gag-pol). 293FT cells were cultured in high glucose Dulbecco's modified Eagle's medium (Invitrogen) with GlutaMAX containing $10 \% \mathrm{FBS}, 100 \mathrm{IU} \mathrm{ml}^{-1}$ penicillin, $100 \mu \mathrm{g} \mathrm{ml}^{-1}$ streptomycin, $25 \mathrm{mM}$ HEPES, $1 \mathrm{mM}$ sodium pyruvate and $0.1 \mathrm{mM}$ non-essential amino acids. Regular media were replaced after $16 \mathrm{~h}$ with growth media containing $10 \mathrm{mM}$ sodium butyrate. After $8 \mathrm{~h}$ of incubation, the medium was changed back to regular growth medium. Twenty-four hours later, the virus-containing supernatants were harvested, filtered through a $0.45-\mu \mathrm{m}$ filter and loaded by centrifugation $(2,000 \mathrm{~g}$, $90 \mathrm{~min}$ at $32^{\circ} \mathrm{C}$ ) on $50 \mu \mathrm{g} \mathrm{ml}^{-1}$ RetroNectin (Takara, Madison, WI) coated nontissue culture six-well plates (Costar). Approximately $2 \times 10^{6}$ cells were added to each well and centrifuged for $20 \mathrm{~min}$ at $600 \mathrm{~g}$, and subsequently maintained at $37^{\circ} \mathrm{C}$ at $5 \% \mathrm{CO}_{2}$ for $48 \mathrm{~h}$ before harvest. For nuclear translocation of oestrogen receptor fusion proteins, 4-OHT was added at a concentration of $500 \mathrm{nM}$.

Cell sorting. For magnetic bead sorting, peripheral blood or BM mononuclear cells were purchased from AllCells LLC and CD19-enrichment was performed by magnetic bead cell sorting according to the manufacturer's instructions (Miltenyi Biotech). Cell sorting by flow cytometry was performed using a BD FACSAriaII 
(BD Biosciences, San Jose, CA). Gating strategies and analysis of the population purity is shown in Supplementary Fig. 1.

Western blotting. After harvesting, the cells were washed twice with PBS and lysed in CelLytic MT buffer (Sigma-Aldrich) supplemented with Mini Complete protease inhibitor (Roche), $1 \%$ phosphatase inhibitor cocktail (Calbiochem) and $1 \mathrm{mM}$ phenylmethylsulphonyl fluoride. After $10 \mathrm{~min}$ incubation on ice and centrifugation at $11,000 \mathrm{~g}$ for $10 \mathrm{~min}$ at $4{ }^{\circ} \mathrm{C}$, the protein concentration was determined by Coomassie Blue Assay (Thermo Scientific). Protein samples were loaded on $4 \%$ to $20 \%$ Bis-Tris gradient gels and transferred on Nitrocellulose membranes (BioRad). The primary antibodies used are listed in Supplementary Table 3. For protein detection, the WesternBreeze immunodetection system (Invitrogen) was used and light emission was either detected by film exposure or by the BioSpectum imaging system (UPV). Original western blots are shown in Supplementary Fig. 6.

Flow cytometry. For analysis of normal B-cell development, BM was stained with the antibodies listed in Supplementary Table 3 and analysed using a BD LSRII Fortessa (BD Biosciences, San Jose, CA). For viability determination by flow cytometry, cells were washed with PBS and resuspended in PBS with 4',6-diamidino-2-phenylindole (Biolegend) or propidium iodide (Sigma-Aldrich) as a marker for dead cells. For detection of apoptotic cells, cells were washed twice in PBS containing $5 \%$ bovine serum albumin (BSA) and stained with Alexa-Fluor 647labelled Annexin V, according to the manufacturer's protocol (Biolegend). For cell cycle analysis, the BrdU Cell Proliferation Assay Kit was purchased from BD Biosciences and performed according to the manufacturer's protocol. In brief, $1 \times 10^{6}$ cells ml $^{-1}$ were cultured for $1 \mathrm{~h}$ in the presence of $1 \mu \mathrm{M} \mathrm{BrdU}$, washed with PBS, fixed and stained according to protocol and analysed by flow cytometry. For the analysis of intracellular ROS formation, ALL cells were incubated for $7 \mathrm{~min}$ with $500 \mathrm{nM} 5$-(and 6-)chloromethyl-2', $7^{\prime}$ dichlorodihydrofluorescein diacetate (CM-H2DCFDA, Invitrogen) at $37^{\circ} \mathrm{C}$ for oxidation of the dye by ROS. After washing with PBS, the cells were incubated for an additional $15 \mathrm{~min}$ at $37^{\circ} \mathrm{C}$ in PBS to allow complete deacetylation of the oxidized form of CM-H2DCFDA by intracellular esterases. The levels of fluorescence were then directly analysed by flow cytometry, gating on viable cells.

Colony-forming assay. The methylcellulose colony-forming assays were performed with 10,000 BCR-ABL1-transformed mouse pre-B ALL cells. Cells were resuspended in mouse MethoCult medium (StemCell Technologies) and cultured on 3-cm diameter dishes, with an extra water supply dish to prevent evaporation. After 21 days, colony numbers were counted by a GelCount analyzer (Oxford Optronix).

Quantitative RT-PCR. Total RNA from cells was extracted using the RNA isolation kit from Macherey Nagel. Complementary DNA was generated with the Vilo SuperScript cDNA synthesis kit (Invitrogen). Quantitative real-time PCR was performed with Fast SYBR Green Master Mix (Invitrogen) and the Viaa7 real-time PCR system (Life Technologies) according to standard PCR conditions. Primer sequences are listed in Supplementary Table 4. For human samples, COX6B was used as a reference gene, for mouse samples Hprt.

Cell viability assay. Imatinib (Novatis Pharmaceuticals, Basel, Switzerland) was reconstituted in distilled water at $10 \mathrm{mM}$ and aliquots were stored at $-20^{\circ} \mathrm{C}$. Thiostrepton was purchased from Sigma, reconstituted in dimethylsulphoxide (DMSO) to $10 \mathrm{mM}$ and stored at $-20^{\circ} \mathrm{C}$. The ARF- and its control peptide were dissolved in DMSO to a final concentration of $10 \mathrm{mM}$ and stored at $-80^{\circ} \mathrm{C}$. $5 \times 10^{4}$ ALL cells were seeded on 96-well plate and drugs were added at indicated concentrations. After 3 days, $15 \mu \mathrm{l}$ of Resazurin (AbD Serotech) were added and cells were incubated for $4 \mathrm{~h}$ before the emission was read at $535 \mathrm{~nm}$ using a Spectramax plate reader (Molecular devices). The fold changes were calculated by using baseline values of untreated cells as a reference.

Single-locus ChIP. Single-locus ChIP was performed as described ${ }^{62}$. In brief, $1 \times 10^{8}$ cells were crosslinked with $1 \%$ formaldehyde for the indicated conditions, and then lysed in $1 \mathrm{ml}$ lysis buffer, and sonicated with six rounds of $30^{\prime \prime}$ on/off, $5 \mathrm{~min}$ each on a bioruptor (Diagenode). The sonication efficacy was determined by resolution on an agarose gel after de-crosslinking. After preclearing with Protein A Agarose (Invitrogen), $5 \mu \mathrm{g}$ per sample FOXM1 antibody (C20-X; Santa Cruz) or normal rabbit IgG (Santa Cruz) was added for overnight $(\mathrm{ON})$ incubation. Then $50 \mu \mathrm{l}$ of $50 \%$ Protein A Agarose slurry was added for $1 \mathrm{~h}$, followed by washing with low salt, high-salt wash buffer and lithium chloride buffer. The DNA was decrosslinked in the presence of $0.2 \mathrm{M} \mathrm{NaCl}$ at $65^{\circ} \mathrm{C} \mathrm{ON}$. RNA was digested and DNA was purified with the Macherey Nagel PCR purification kit. PCR was performed as described in the section for quantitative real-time PCR. A list of primers is provided in Supplementary Table 4.

Immunofluorescence analysis. Cells were seeded onto retronectin-coated chamber slides and treated with 5 and $10 \mu \mathrm{M}$ ARF peptide for $18 \mathrm{~h}$. Cells were fixed with $4 \%$ paraformaldehyde for $10 \mathrm{~min}$ at room temperature (RT), permeabilized in PBS/0.15\% Triton-X-100 for 2 min at RT and blocked in 1\% BSA/PBS for $1 \mathrm{~h}$ at RT. Slides were then incubated with antibodies against fibrillarin (clone $38 \mathrm{~F} 3$, ab4566, 1/250) and FOXM1 (clone D12D5, CST\#5436, 1/50) in 1\% BSA/PBS $\mathrm{ON}$ at $4{ }^{\circ} \mathrm{C}$. Slides were washed three times for $5 \mathrm{~min}$ in PBS and incubated for $1 \mathrm{~h}$ at RT in $1 \%$ BSA/PBS with Alexa Fluor 488-conjugated goat anti-mouse (Molecular Probes, A-11029) and Alexa Fluor 594-conjugated goat anti-rabbit (Molecular Probes, A-11037) secondary antibodies. Slides were then washed three times for $5 \mathrm{~min}$ in PBS and mounted using Vectashield (Vector Laboratories) containing $1 \mu \mathrm{g} \mathrm{ml}^{-1} 4^{\prime}$,6-diamidino-2-phenylindole. Images were acquired on a Leica SP5 upright confocal microscope and processed using Volocity software (Perkin Elmer).

In vivo leukaemia cell transplantation. All mouse experiments were subject to institutional approval by the University of California San Francisco Institutional Animal Care and Use Committee. BCR-ABL1-transformed murine Foxm $\mathrm{f}^{\mathrm{fl} / \mathrm{fl}}$ pre-B ALL cells transduced with either empty vector (EV, $\mathrm{ER}^{\mathrm{T} 2}$ without Cre) or Cre (Cre$\mathrm{ER}^{\mathrm{T} 2}$ ) containing a puromycin resistance cassette. After selection for 2 days with $2 \mu \mathrm{g} \mathrm{ml}^{-1}$ puromycin, successful selection was verified by fluorescence-activated cell sorting analysis (no living cell in untransduced control cells) and subsequently grown in vitro for expansion of cell numbers. Experiments were performed within 4 weeks after BM harvest to avoid secondary mutations. Ex vivo deletion of Foxm 1 was induced in the Cre- $\mathrm{ER}^{\mathrm{T} 2}$ group by adding 4-OHT $24 \mathrm{~h}$ before transplantation. To rule out the possible homing defects after Foxm1 deletion as a potential confounding variable, cells were injected intrafemorally into sublethally irradiated $(2.5 \mathrm{~Gy}) \mathrm{NOD} /$ SCID mice. Seven mice per group were injected. For in vivo deletion of Foxm1, 100,000 BCR-ABL1-transformed murine Foxm $1^{\mathrm{f} / \mathrm{fl}}$ pre-B ALL cells with either EV or Cre were injected i.v. and deletion induced by tamoxifen injection daily at $0.4 \mathrm{mg}$ per mouse for 10 days. Terminally ill mice were killed and spleen and/or BM harvested for further analysis. For treatment of human xenografts, LAX7R cells were transduced with a luc-GFP vector and sorted for GFP expression. Cells $\left(5 \times 10^{5}\right)$ were injected i.v. into NSG mice (NOD/SCID-IL2 receptor gamma chain knockout), seven mice per group and treatment was initiated. ARF peptide was administered i.v. and i.p. at $10 \mathrm{mg} \mathrm{kg}^{-1}$ daily (total dose $20 \mathrm{mg} \mathrm{kg}^{-1}$ ) for 10 consecutive days and Thiostrepton i.v. at $50 \mathrm{mg} \mathrm{kg}^{-1}$ daily for seven consecutive days. ARF peptide was resolved fresh every day to avoid freeze-thawing steps. C-terminal amidation and $\mathrm{N}$-terminal acetylation was used to increase peptide half-life in vivo. Thiostrepton was resolved at $25 \mathrm{mg} \mathrm{ml}^{-1}$ in DMSO and diluted in PBS before injection. To increase solubility, $2 \mathrm{~min}$ of sonication was performed daily before injection. Bioimaging of leukaemia burden in the mice was performed at day 14 using an in vivo IVIS 100 bioluminescence/optical imaging system (Xenogen). D-Luciferin (Promega) was injected intraperitoneally at a dose of $2.5 \mathrm{mg}$ per mouse in PBS $12 \mathrm{~min}$ before measuring the luminescence signal. General anaesthesia was induced with $5 \%$ isoflurane and continued during the procedure with $2 \%$ isoflurane introduced through a nose cone. When a mouse became terminally sick, it was killed, and BM and/or spleen were collected for further analysis.

\section{References}

1. Chen, X. et al. The forkhead transcription factor FOXM1 controls cell cycledependent gene expression through an atypical chromatin binding mechanism Mol. Cell. Biol. 33, 227-236 (2013).

2. Chen, Y. J. et al. A conserved phosphorylation site within the forkhead domain of FoxM1B is required for its activation by cyclin-CDK1. J. Biol. Chem. 284, 30695-30707 (2009).

3. Laoukili, J. et al. Activation of FoxM1 during G2 requires cyclin A/Cdkdependent relief of autorepression by the FoxM1 N-terminal domain. Mol. Cell. Biol. 28, 3076-3087 (2008).

4. Park, H. J. et al. An N-terminal inhibitory domain modulates activity of FoxM1 during cell cycle. Oncogene 27, 1696-1704 (2008).

5. Wang, I. C. et al. Forkhead box M1 regulates the transcriptional network of genes essential for mitotic progression and genes encoding the SCF (Skp2Cks1) ubiquitin ligase. Mol. Cell. Biol. 25, 10875-10894 (2005).

6. Alvarez-Fernandez, M. et al. Recovery from a DNA-damage-induced G2 arrest requires Cdk-dependent activation of FoxM1. EMBO Rep. 11, 452-458 (2010).

7. Park, H. J. et al. FoxM1, a critical regulator of oxidative stress during oncogenesis. EMBO J. 28, 2908-2918 (2009).

8. Wierstra, I. FOXM1 (Forkhead box M1) in tumorigenesis: overexpression in human cancer, implication in tumorigenesis, oncogenic functions, tumorsuppressive properties, and target of anticancer therapy. Adv. Cancer Res. 119, 191-419 (2013).

9. Gusarova, G. A. et al. A cell-penetrating ARF peptide inhibitor of FoxM1 in mouse hepatocellular carcinoma treatment. J. Clin. Invest. 117, 99-111 (2007).

10. Kalinichenko, V. V. et al. Foxmlb transcription factor is essential for development of hepatocellular carcinomas and is negatively regulated by the p19ARF tumor suppressor. Genes Dev. 18, 830-850 (2004). 
11. Yoshida, Y., Wang, I. C., Yoder, H. M., Davidson, N. O. \& Costa, R. H. The forkhead box M1 transcription factor contributes to the development and growth of mouse colorectal cancer. Gastroenterology 132, 1420-1431 (2007).

12. Kim, I. M. et al. The Forkhead Box $\mathrm{ml}$ transcription factor stimulates the proliferation of tumor cells during development of lung cancer. Cancer Res. 66, 2153-2161 (2006).

13. Wang, I. C. et al. Deletion of Forkhead Box M1 transcription factor from respiratory epithelial cells inhibits pulmonary tumorigenesis. PLOS ONE 4, e6609 (2009).

14. Wang, I. C. et al. Transgenic expression of the forkhead box M1 transcription factor induces formation of lung tumors. Oncogene 27, 4137-4149 (2008).

15. Kalin, T. V. et al. Increased levels of the FoxM1 transcription factor accelerate development and progression of prostate carcinomas in both TRAMP and LADY transgenic mice. Cancer Res. 66, 1712-1720 (2006).

16. Nakamura, S. et al. The FOXM1 transcriptional factor promotes the proliferation of leukemia cells through modulation of cell cycle progression in acute myeloid leukemia. Carcinogenesis 31, 2012-2021 (2010).

17. Park, Y. Y. et al. FOXM1 mediates Dox resistance in breast cancer by enhancing DNA repair. Carcinogenesis 33, 1843-1853 (2012).

18. Sanders, D. A., Ross-Innes, C. S., Beraldi, D., Carroll, J. S. \& Balasubramanian, $\mathrm{S}$. Genome-wide mapping of FOXM1 binding reveals co-binding with estrogen receptor alpha in breast cancer cells. Genome Biol. 14, R6 (2013).

19. Uddin, S. et al. Overexpression of FoxM1 offers a promising therapeutic target in diffuse large B-cell lymphoma. Haematologica 97, 1092-1100 (2012).

20. Wang, X., Kiyokawa, H., Dennewitz, M. B. \& Costa, R. H. The Forkhead Box $\mathrm{m} 1 \mathrm{~b}$ transcription factor is essential for hepatocyte DNA replication and mitosis during mouse liver regeneration. Proc. Natl Acad. Sci. USA 99, 16881-16886 (2002)

21. Carr, J. R., Park, H. J., Wang, Z., Kiefer, M. M. \& Raychaudhuri, P. FoxM1 mediates resistance to herceptin and paclitaxel. Cancer Res. 70, 5054-5063 (2010).

22. Inaba, H., Greaves, M. \& Mullighan, C. G. Acute lymphoblastic leukaemia. Lancet 381, 1943-1955 (2013).

23. Lee, H. J., Thompson, J. E., Wang, E. S. \& Wetzler, M. Philadelphia chromosome-positive acute lymphoblastic leukemia: current treatment and future perspectives. Cancer 117, 1583-1594 (2011).

24. Pui, C. H., Relling, M. V. \& Downing, J. R. Acute lymphoblastic leukemia. N. Engl. J. Med. 350, 1535-1548 (2004).

25. Fielding, A. K. et al. UKALLXII/ECOG2993: addition of imatinib to a standard treatment regimen enhances long-term outcomes in Philadelphia positive acute lymphoblastic leukemia. Blood 123, 843-850 (2013).

26. Druker, B. J. et al. Effects of a selective inhibitor of the Abl tyrosine kinase on the growth of Bcr-Abl positive cells. Nat. Med. 2, 561-566 (1996).

27. Druker, B. J. et al. Activity of a specific inhibitor of the BCR-ABL tyrosine kinase in the blast crisis of chronic myeloid leukemia and acute lymphoblastic leukemia with the Philadelphia chromosome. New Engl. J. Med. 344, 1038-1042 (2001).

28. van Zelm, M. C. et al. Ig gene rearrangement steps are initiated in early human precursor B cell subsets and correlate with specific transcription factor expression. J. Immunol. 175, 5912-5922 (2005).

29. Hardy, R. R., Carmack, C. E., Shinton, S. A., Kemp, J. D. \& Hayakawa, K. Resolution and characterization of pro-B and pre-pro-B cell stages in normal mouse bone marrow. J. Exp. Med. 173, 1213-1225 (1991).

30. Hobeika, E. et al. Testing gene function early in the B cell lineage in mb1-cre mice. Proc. Natl Acad. Sci. USA 103, 13789-13794 (2006).

31. Bodine, D. M., Karlsson, S. \& Nienhuis, A. W. Combination of interleukins 3 and 6 preserves stem cell function in culture and enhances retrovirus-mediated gene transfer into hematopoietic stem cells. Proc. Natl Acad. Sci. USA 86, 8897-8901 (1989).

32. Dai, Y., Rahmani, M., Corey, S. J., Dent, P. \& Grant, S. A Bcr/Abl-independent, Lyn-dependent form of imatinib mesylate (STI-571) resistance is associated with altered expression of Bcl-2. J. Biol. Chem. 279, 34227-34239 (2004).

33. Janes, M. R. et al. Effective and selective targeting of leukemia cells using a TORC1/2 kinase inhibitor. Nat. Med. 16, 205-213 (2010).

34. Karadedou, C. T. et al. FOXO3a represses VEGF expression through FOXM1dependent and -independent mechanisms in breast cancer. Oncogene 31, 1845-1858 (2012).

35. McGovern, U. B. et al. Gefitinib (Iressa) represses FOXM1 expression via FOXO3a in breast cancer. Mol. Cancer Ther. 8, 582-591 (2009).

36. Lam, E. W., Brosens, J. J., Gomes, A. R. \& Koo, C. Y. Forkhead box proteins: tuning forks for transcriptional harmony. Nat. Rev. Cancer 13, 482-495 (2013)

37. Kikuchi, S. et al. Active FKHRL1 overcomes imatinib resistance in chronic myelogenous leukemia-derived cell lines via the production of tumor necrosis factor-related apoptosis-inducing ligand. Cancer Sci. 98, 1949-1958 (2007).

38. Chelikani, P., Fita, I. \& Loewen, P. C. Diversity of structures and properties among catalases. Cell. Mol. Life Sci. 61, 192-208 (2004).
39. Lieberthal, W., Triaca, V., Koh, J. S., Pagano, P. J. \& Levine, J. S. Role of superoxide in apoptosis induced by growth factor withdrawal. Am. J. Physiol. 275, F691-F702 (1998).

40. Tan, W. Q., Wang, K., Lv, D. Y. \& Li, P. F. Foxo3a inhibits cardiomyocyte hypertrophy through transactivating catalase. J. Biol. Chem. 283, 29730-29739 (2008).

41. Song, Z. \& Wu, M. Identification of a novel nucleolar localization signal and a degradation signal in Survivin-deltaEx3: a potential link between nucleolus and protein degradation. Oncogene 24, 2723-2734 (2005).

42. Bertwistle, D., Sugimoto, M. \& Sherr, C. J. Physical and functional interactions of the Arf tumor suppressor protein with nucleophosmin/B23. Mol. Cell. Biol. 24, 985-996 (2004).

43. Itahana, K. et al. Tumor suppressor ARF degrades B23, a nucleolar protein involved in ribosome biogenesis and cell proliferation. Mol. Cell 12, 1151-1164 (2003).

44. Hegde, N. S., Sanders, D. A., Rodriguez, R. \& Balasubramanian, S. The transcription factor FOXM1 is a cellular target of the natural product thiostrepton. Nat. Chem. 3, 725-731 (2011).

45. Xie, Z. et al. Foxm1 transcription factor is required for maintenance of pluripotency of P19 embryonal carcinoma cells. Nucleic Acids Res. 38, 8027-8038 (2010).

46. Bhat, U. G., Halasi, M. \& Gartel, A. L. Thiazole antibiotics target FoxM1 and induce apoptosis in human cancer cells. PLoS ONE 4, e5592 (2009).

47. Bhat, U. G., Zipfel, P. A., Tyler, D. S. \& Gartel, A. L. Novel anticancer compounds induce apoptosis in melanoma cells. Cell Cycle 7, 1851-1855 (2008).

48. Halasi, M. \& Gartel, A. L. A novel mode of FoxM1 regulation: positive autoregulatory loop. Cell Cycle 8, 1966-1967 (2009).

49. Sattler, M. et al. The BCR/ABL tyrosine kinase induces production of reactive oxygen species in hematopoietic cells. J. Biol. Chem. 275, 24273-24278 (2000)

50. Ozben, T. Oxidative stress and apoptosis: impact on cancer therapy. J. Pharm. Sci. 96, 2181-2196 (2007).

51. DeNicola, G. M. et al. Oncogene-induced Nrf2 transcription promotes ROS detoxification and tumorigenesis. Nature 475, 106-109 (2011).

52. Cholez, E. et al. Evidence for a protective role of the STAT5 transcription factor against oxidative stress in human leukemic pre-B cells. Leukaemia. 26, 2390-2397 (2012).

53. Casetti, L. et al. Differential contributions of STAT5A and STAT5B to stress protection and tyrosine kinase inhibitor resistance of chronic myeloid leukemia stem/progenitor cells. Cancer Res. 73, 2052-2058 (2013).

54. Juric, D. et al. Differential gene expression patterns and interaction networks in BCR-ABL-positive and -negative adult acute lymphoblastic leukemias. J Clin. Oncol. 25, 1341-1349 (2007).

55. Hogan, L. E. et al. Integrated genomic analysis of relapsed childhood acute lymphoblastic leukemia reveals therapeutic strategies. Blood 118, 5218-5226 (2011).

56. Konig, H. et al. Enhanced Bcr-Abl-specific antileukemic activity of arsenic trioxide (Trisenox) through glutathione-depletion in imatinib-resistant cells. Haematologica 92, 838-841 (2007).

57. Kwee, J. K. et al. Modulation of reactive oxygen species by antioxidants in chronic myeloid leukemia cells enhances imatinib sensitivity through survivin downregulation. Anticancer Drugs 19, 975-981 (2008).

58. Gormally, M. V. et al. Suppression of the FOXM1 transcriptional programme via novel small molecule inhibition. Nat. Commun. 5, 5165 (2014).

59. Zhang, Y., Xiong, Y. \& Yarbrough, W. G. ARF promotes MDM2 degradation and stabilizes p53: ARF-INK4a locus deletion impairs both the Rb and p53 tumor suppression pathways. Cell 92, 725-734 (1998).

60. Kirschner-Schwabe, R. et al. Expression of late cell cycle genes and an increased proliferative capacity characterize very early relapse of childhood acute lymphoblastic leukemia. Clin. Cancer Res. 12, 4553-4561 (2006).

61. Pawitan, Y. et al. Gene expression profiling spares early breast cancer patients from adjuvant therapy: derived and validated in two population-based cohorts. Breast Cancer Res. 7, R953-R964 (2005).

62. Reynaud, D. et al. Regulation of B cell fate commitment and immunoglobulin heavy-chain gene rearrangements by Ikaros. Nat. Immunol. 9, 927-936 (2008).

\section{Acknowledgements}

We thank Dr Hassan Jumaa, Ulm, Germany and Dr Kevin Shannon, San Francisco, CA for critical discussions. This work was supported by grants from the NIH/NCI through R01CA137060, R01CA139032, R01CA169458, R01CA172558 and R01CA157644 (to M.M.), grants from the Leukemia and Lymphoma Society (to M.M.), the California Institute for Regenerative Medicine through TR02-1816 (M.M.), the William Lawrence and Blanche Hughes Foundation and a Leukaemia Lymphoma Research project grant (to M.M.). M.M. is a Scholar of The Leukemia and Lymphoma Society and a Wellcome Trust Senior Investigator. P.R. is supported by NCI grants CA175380 and CA177655. 


\section{Author contributions}

M.B. and M.M. conceived the study, designed experiments and interpreted the data. M.B. performed majority of the experiments. E. Park and J.F. performed the experiments. H.G. generated analyses for clinical patient data. E. Passegué, L.K., H.S., A.M., E. Paietta, D.K. and P.R. provided important reagents, mouse samples and patient data. M.B. and M.M. wrote the manuscript.

\section{Additional information}

Supplementary Information accompanies this paper at http://www.nature.com/ naturecommunications

Competing financial interests: There are no competing financial interests.
Reprints and permission information is available online at http://npg.nature.com/ reprintsandpermissions/

How to cite this article: Buchner, M. et al. Identification of FOXM1 as a therapeutic target in B-cell lineage acute lymphoblastic leukaemia. Nat. Commun. 6:6471 doi: 10.1038/ncomms7471 (2015).

This work is licensed under a Creative Commons Attribution 4.0 International License. The images or other third party material in this article are included in the article's Creative Commons license, unless indicated otherwise in the credit line; if the material is not included under the Creative Commons license, users will need to obtain permission from the license holder to reproduce the material. To view a copy of this license, visit http://creativecommons.org/licenses/by/4.0/ 